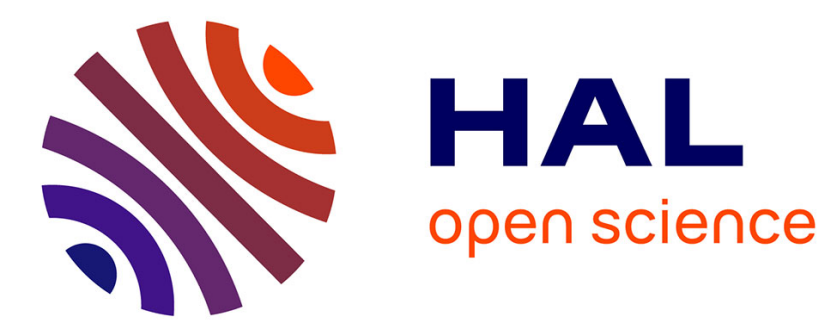

\title{
Asteroid shape and spin statistics from convex models
}

\author{
J. Torppa, V.-P. Hentunen, P. Pääkkönen, P. Kehusmaa, K. Muinonen
}

\section{To cite this version:}

J. Torppa, V.-P. Hentunen, P. Pääkkönen, P. Kehusmaa, K. Muinonen. Asteroid shape and spin statistics from convex models. Icarus, 2008, 198 (1), pp.91. 10.1016/j.icarus.2008.07.014. hal00499092

\section{HAL Id: hal-00499092 \\ https://hal.science/hal-00499092}

Submitted on 9 Jul 2010

HAL is a multi-disciplinary open access archive for the deposit and dissemination of scientific research documents, whether they are published or not. The documents may come from teaching and research institutions in France or abroad, or from public or private research centers.
L'archive ouverte pluridisciplinaire HAL, est destinée au dépôt et à la diffusion de documents scientifiques de niveau recherche, publiés ou non, émanant des établissements d'enseignement et de recherche français ou étrangers, des laboratoires publics ou privés. 


\section{Accepted Manuscript}

Asteroid shape and spin statistics from convex models

J. Torppa, V.-P. Hentunen, P. Pääkkönen, P. Kehusmaa, K. Muinonen

PII:

S0019-1035(08)00283-2

DOI: $\quad$ 10.1016/j.icarus.2008.07.014

Reference: $\quad$ YICAR 8734

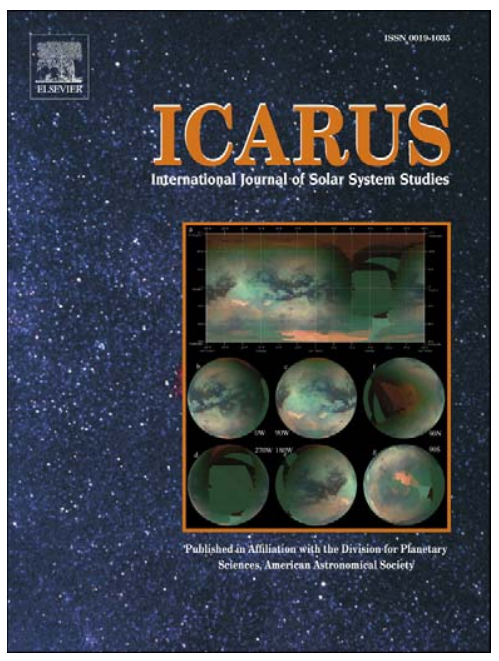

To appear in: Icarus

Received date: 18 September 2007

Revised date: 3 July 2008

Accepted date: 7 July 2008

Please cite this article as: J. Torppa, V.-P. Hentunen, P. Pääkkönen, P. Kehusmaa, K. Muinonen, Asteroid shape and spin statistics from convex models, Icarus (2008), doi: 10.1016/j.icarus.2008.07.014

This is a PDF file of an unedited manuscript that has been accepted for publication. As a service to our customers we are providing this early version of the manuscript. The manuscript will undergo copyediting, typesetting, and review of the resulting proof before it is published in its final form. Please note that during the production process errors may be discovered which could affect the content, and all legal disclaimers that apply to the journal pertain. 


\title{
Asteroid shape and spin statistics from convex models
}

\author{
J. Torppa ${ }^{1}$ \\ Observatory, P.O.Box 14, 00014 University of Helsinki, Finland \\ V-P. Hentunen \\ Taurus Hill Observatory, Kangaslampi, Finland \\ P. Pääkkönen \\ Jakokoski Observatory, Jakokoski, Finland \\ P. Kehusmaa \\ Slope Rock Observatory, Hyvinkää, Finland \\ K. Muinonen \\ Observatory, P.O.Box 14, 00014 University of Helsinki, Finland
}

Number of manuscript pages $=55$

Number of figures $=19$

Number of tables $=4$

\footnotetext{
${ }^{1}$ email:johanna.torppa@helsinki.fi
} 
Running head: Asteroid spin and shape statistics

Editorial correspondence and proofs:

Johanna Torppa

johanna.torppa@helsinki.fi

Observatory

PB 14

00014 University of Helsinki

Finland

tel:+358-50-3259315 


\begin{abstract}
We introduce techniques for characterizing convex shape models of asteroids with a small number of parameters, and apply these techniques to a set of 87 models from convex inversion. We present three different approaches for determining the overall dimensions of an asteroid. With the first technique, we measured the dimensions of the shapes in the direction of the rotation axis and in the equatorial plane and with the two other techniques, we derived the best-fit ellipsoid. We also computed the inertia matrix of the model shape to test how well it represents the target asteroid, i.e., to find indications of possible non-convex features or albedo variegation, which the convex shape model cannot reproduce. We used shape models for 87 asteroids to perform statistical analyses and to study dependencies between shape and rotation period, size, and taxonomic type. We detected correlations, but more data are required, especially on small and large objects, as well as slow and fast rotators, to reach a more thorough understanding about the dependencies. Results show, e.g., that convex models of asteroids are not that far from ellipsoids in root-mean-square sense, even though clearly irregular features are present. We also present new spin and shape solutions for asteroids (31) Euphrosyne, (54) Alexandra, (79) Eurynome, (93) Minerva, (130) Elektra, (376) Geometria, (471) Papagena, and (776) Berbericia. We used a socalled semi-statistical approach to obtain a set of possible spin state solutions. The number of solutions depends on the abundancy of the data, which for Eurynome, Elektra, and Geometria was extensive enough for determining an unambiguous spin and shape solution. Data of Euphrosyne, on the other hand, provided a wide distribution of possible spin solutions, whereas the rest of the targets have two or three possible solutions.
\end{abstract}

Key words: asteroids, shapes, spins 


\section{Introduction}

Traditionally, asteroid shapes have been modelled using triaxial ellipsoids, which are completely defined by only two parameters, namely, the ratios of the lengths of the principal axes. Lately, complex shape models, such as arbitrary polyhedra and even non-convex shapes, have become common, raising a question about how to describe the characteristic features of the models. One of the lightcurve inversion methods developed in the $20^{\text {th }}$ century is the convex inversion method (Kaasalainen and Torppa 2001 and Kaasalainen et al. 2001, hereafter KT01 and K01, respectively), which offers an arbitrary polyhedron shape model. Also, radar and even spacecraft-based non-convex models have become more and more common (e.g., Magri et al. 2007 and Demura et al. 2006, respectively). Although these models are naturally much more informative than ellipsoids, their weakness is that there is no way to express the exact shape solution in a simple numerical form due to the large number of parameters. We can, however, characterize the shape using various quantities, and grasp the most important shape features with a small number of parameters, which is required, e.g., when carrying out statistical analyses of the shape characteristics.

Previous studies considering spin states and shapes of asteroids in statistical sense have used triaxial ellipsoid shape models. Ellipsoid dimensions of 22 main-belt asteroids were determined by Magnusson (1990) who conclude, based on the triaxial models, that it is not likely that centrifugal forces have affected the shapes. On the other hand, Drummond et al. (1991) studied the axis distributions of 26 asteroids concluding that shapes of some of the objects may be jacobi ellipsoids, i.e., the stretching of the ellipsoids is due to the rotation of the body. Hartmann et al. (1988) compared the axis ratios of a sample of 26 Trojan and Hilda asteroids to those of main-belt asteroids. They suggest, that Trojans and Hildas are more elongated that main-belt asteroids of comparable size.

Shape characterization is an important part in many branches of science, e.g., in small- 


\section{ACCEPTED MANUSCRIPT}

particle analysis for which commercial image analyzers have already been developed. The image of the particle is often projected to a plane and, hence, the parameters describe the two-dimensional projection of the shape. These parameters usually include the perimeter, area, and maximum and minimum dimensions which are compared to those of a circle with the same area or circumference, or to the convex hull of the particle to define its roundedness, convexity, or dimensions. One example of small particle shape determination is the shape analysis of lunar dust particles as described in Liu et al. (2007), who used 2-D images of the sample particles to define their areas and perimeters, and the circular-equivalent diameter from the measured areas. They also fit ellipses to the measured areas in the least-squares sense. Fractal analysis for particle shape characterization has been applied by Maria and Carey (2002) when analyzing the shapes of volcanic particles. Muinonen (2006) presents a method to derive statistical particle shape paramters from a set of 2-D particle silhouettes using a Gaussian shape model consisting of two parameters. A method to characterize macroscopic objects is presented, e.g., by Torppa et al. (2006), who determined the characteristic shape parameters of potato tubers. They used 3-D image data to find the ellipsoid that best fits the tuber shape. They also used the residual radii of the ellipsoid fits in a statistical sense to determine the variance of the Gaussian distribution describing the local irregular surface features. Gaussian statistics have been applied to asteroid shape characterization also by Muinonen and Lagerros (1998), who determined the parameters for the Gaussian random sphere that best mimics asteroids and other small bodies.

In this study, we introduce and compare techniques to express the overall dimensions and irregularity of an arbitrary convex polyhedron and apply the techniques to a set of 87 shape models for studying the statistics of basic shape features of asteroids. We also present new spin and shape results for eight asteroids of which new lightcurve observations have been carried out.

In Secs. 2 and 3, we describe the methods used in lightcurve inversion and characteri- 
zation of arbitrary shapes. Section 4 is dedicated to results and Sec. 5 to conclusions and future prospects.

\section{Convex inversion for spins and shapes}

Spin and shape analysis for selected targets with new photometric observations was carried out using the convex inversion method. Theory of the method is described in detail in KT01 and K01, and is not repeated here. In the following, however, we consider a few developments.

One fact that has not been discussed in the earlier papers is the number of parameters (i.e., facets) in the Minkowski minimization, which is used for determining the polyhedron vertices from the set of facet normals. It has been mentioned in previous papers (e.g., KT01) that, when searching for the best-fit facet areas, the number of facets in the polyhedron shape model has to be of order 800 to guarantee that the shape solution does not depend on the choice of the facet normal directions. In the next step of finding the vertices of the shape from the facet information, however, the number of facets can be significantly reduced. Without affecting the final shape, this recombination significantly speeds up the Minkowski minimization which previously was the most time consuming part of the procedure. We found it most convenient to combine the facets around each vertex determined from the standard triangulation. Normal directions of the combined facets are calculated by taking a weighted mean of the original normal directions. Due to the way of discretization and combination, some facets remain uncombined, but this does not affect the final shape solution.

Another change to the inversion procedure was that, from the beginning, we aimed at a distribution of spin solutions, not at a single unambiguous solution, since the data sets were not as extensive as in the previous analyses (e.g., Torppa et al., 2003; hereafter T03). We call this technique semi-statistical, since we obtain the distribution of possible solutions, but 


\section{ACCEPTED MANUSCRIPT}

not the probability density. As before, we carried out the initial spin state determination by scanning through a set of spin axis directions and rotation periods, while fitting a low-order functional series shape model. The time step of the period scanning was $\Delta P / 4$, where

$$
\Delta P=\frac{P^{2}}{2 T}
$$

$P$ being the period, and $T$ the total time span of the observations. This equation is based on the fact that, if $P$ is changed by $\Delta P$, the lightcurve is phase-shifted by $\pi \operatorname{during} T$. Thus, if we have model and data lightcurves with two equal maxima and minima (typical for an ellipsoid) of which the first and the last coincide for period $P$, then they coincide also for period $P+\Delta P$. Although the data lightcurves usually differ significantly from those produced by an ellipsoid, this equation gives a rough estimate about how densely the $\chi^{2}$ minima are located in the $\chi^{2}, P$-space, and how densely $P$ should be sampled. Spin axis was sampled in eight uniformly distributed directions. From the initial spin state search, we accepted all the solutions with $r m s<\min (r m s) \times 1.5$, which is an empirical criterion for finding the final solution or solutions from the set of initial ones. Starting from the set of initial solutions, we refined each by adding more shape parameters (a higher-order functional series) and setting the spin axis direction and rotation period as free parameters. For the improved solutions, we finally applied the standard Gaussian distribution empirical rule for 3-sigma deviation as the criterion of acceptance. Due to the effects of the uncertainties in the shape and scattering models, an error of $5^{\circ}$ should be added to each spin longitude and $15^{\circ}$ to each latitude. This uncertainty is an empirical value, based on this study and earlier applications (e.g., T03).

The last aspect to consider was the sampling density of the data. Since some of the lightcurves are over-densely sampled, while others are sparse, the weight of each data point could be set inversely proportional to the lightcurve sampling density in order to prevent 
dense lightcurves from dominating in the $\chi^{2}$ computation. The function to be minimized would then be

$$
\chi^{2}=\sum_{i} \frac{\left(L_{d, i}-L_{m, i}\right)^{2}}{\sigma_{i}^{2}}
$$

where $L_{d, i}$ and $L_{m, i}$ are the corresponding data and model brightnesses. $\sigma_{i}^{2}$ is proportional to the number of data points in and inversely proportional to the overall duration of the $i^{\text {th }}$ lightcurve.

\section{Shape characterization}

The definition of dimensions of convex inversion models is not straightforward, since they are irregular, and not necessarily in the principal-axis rotation state. In the previous papers, where convex inversion has beed applied to asteroid lightcurve data (e.g., Kaasalainen et al., 2002a and T03), descriptive axis ratios for the model shapes are given as the mean of two measures: the axis ratios computed from the largest dimensions in the rotation axis direction and in the equatorial plane (hereafter the OD technique, section 3.1 of this paper), and the axis ratios of the best-fit ellipsoid as defined in section 3.2 of this paper (hereafter the E1 technique). There are, however, also many other ways to define the dimensions. In this paper, we compare four approaches to characterize the shape of an arbitrary convex polyhedron. Before presenting the techniques, we discuss the rotational state of the shape models, since the models are rarely in the exact principal-axis rotation state, although the target asteroids with only one lightcurve period should be. When finding the characteristic shape features, it is not clear whether the body should be rotated to the principal-axis state. Here we include both approaches: methods that fix one axis to be aligned with the rotation axis and ones that allow the coordinate system to be rotated arbitrarily. In the first two methods, i.e., the one finding the overall dimensions of the shape (the OD technique) and 
the one defining the best-fit ellipsoid in the sense of facet distances (the E1 technique), the model shape is not rotated to the principal-axis state. The third technique, that gives the best-fit ellipsoid in the sense of radii lengths (hereafter the E2 technique, section 3.3) the shortest axis is not forced to be aligned with the rotation axis of the model. Thus, only the shape is fitted, disregarding the rotational state. The fourth technique, computation of the principal moments of inertia (hereafter the PMI technique, section 3.4), also considers only the shape without fixing the original rotation state.

\subsection{Overall dimensions (OD)}

The overall dimensions of the shape were computed in three stages (Fig. 1). First, the largest extent in z-axis (spin axis) direction was calculated from

$$
2 c=\max \left(z_{i}\right)-\min \left(z_{i}\right)
$$

where $z_{i}$ are the $z$ coordinates of all the polyhedron vertices. Second, the largest extent of projection to $x y$-plane was found from

$$
2 a=\max \left(x_{i} \cos \phi_{a x}+y_{i} \sin \phi_{a x}\right)-\min \left(x_{i} \cos \phi_{a x}+y_{i} \sin \phi_{a x}\right),
$$

where $x_{i}$ and $y_{i}$ are the values of the $x$ and $y$ coordinates of all the polyhedron vertices. $\phi_{a x}$ is the azimuthal angle between $x$-axis and the trial direction in which the extent of the projection was calculated. $\phi_{a x}$ was sampled with one degree step over the range $\left[0^{\circ}, 180^{\circ}\right]$. Last, the largest extent, $2 b$, of projection to $x y$-plane, perpendicular to direction of $a$, was computed analogously with $2 a$.

Figure 1 


\subsection{Ellipsoid I (E1)}

With the first of the ellipsoid-based techniques, we compare the support functions of the model and the ellipsoid. The support function is defined as

$$
\rho(\theta, \phi)=\mathbf{n}(\theta, \phi) \cdot \mathbf{r}(\theta, \phi)
$$

where $\theta$ and $\phi$ are the spherical coordinates, $\mathbf{n}$ is the surface normal, and $\mathbf{r}$ is the radius vector. Thus, $\rho(\theta, \phi)$ is the distance of the tangent plane at point $(\theta, \phi)$ from the origin. As in Kaasalainen et al. (1992), we calculated the difference

$$
d_{f}=\sum_{i} \frac{\left|\rho_{m, i}-\rho_{e, i}\right|}{\rho_{e, i}} g_{i}
$$

where $g_{i}$ is the area of the corresponding facet element on a unit sphere, $\rho_{m, i}$ is the distance

of the model shape's facet $i$ from the origin, and $\rho_{e, i}$ is the distance of corresponding ellipsoid facet (with the same surface normal) from the origin.

We chose the orientation (origin and $z$-axis) of the ellipsoid to coincide with that of the model. The ellipsoid axis ratios were searched by setting the shortest (polar) axis length $c=1$, and sampling the azimuthal axis lengths $a, b$ within the range $[1,3]$, with a step $\delta=0.1$. Axes were then scaled so that the ellipsoid and the model were of the same volume. The azimuthal rotation $\phi_{a x}$ of the longest ellipsoid $a$-axis with respect to the $x$-axis was sampled over the range $\left[0^{\circ}, 90^{\circ}\right]$ with a step of $\delta=10^{\circ}$.

\subsection{Ellipsoid II (E2)}

Another way of finding the characteristic ellipsoid dimensions is to compare the radii of the model shape and the ellipsoid. We did not force ellipsoid z-axis to coincide with model's rotation axis or ellipsoid origin with that of the model, and minimized function $\chi_{e}^{2}$ of nine 
free parameters: semi-axis of the ellipsoid $(a, b, c)$, transformation of the origin of the model shape $\left(\mathbf{r}_{c}\right)$ to coincide with the origin of the ellipsoid, and rotation of the model shape $(\alpha, \beta$, and $\gamma$ ) for its axes to coincide with the principal axes of the ellipsoid:

$$
\chi_{e}^{2}=\sum_{i} \frac{\left(r_{m, i}-r_{e, i}\right)^{2}}{\bar{r}_{m, i}^{2}}
$$

where $r_{m, i}$ is the model shape's $i^{\text {th }}$ radius in a system whose origin translated by $\mathbf{r}_{c}$ and which is rotated by Euler angles $\alpha, \beta$, and $\gamma$, and $\bar{r}_{m, i}$ is the mean of the model radii. $r_{e, i}$ are the ellipsoid radii calculated for the same vertex directions as the model radii $r_{m, i}$. The minimization was carried out using Nelder and Mead's simplex method described in Press et al. (1994).

\subsection{Principal moments of inertia (PMI)}

The inertia tensor of an arbitrary shape can be calculated by summing up the moments and products of inertia for different parts of the shape. In this case, we found it most practical to divide each shape into polygonal cones and furthermore into tetrahedra so that the total inertia matrix is obtained from

$$
I_{j k}=\sum_{i} t_{j k, i}
$$

where $t_{j k, i}$ is the moment $(j=k)$ or product $(j \neq k)$ of inertia of a single tetrahedron. $j, k=[1,3]$ correspond to the $x, y$, and $z$ coordinates and $i$ stands for the $i^{\text {th }}$ tetrahedron. For calculating $t_{i}$, we followed the formulas given by Tonon (2004). The principal moments and principal axes were obtained by diagonalizing the inertia tensor $I$ using Jacobi transformations (Press et al., 1994). 


\section{Results}

\subsection{New spin and shape solutions}

New spin and shape solutions are presented for eight asteroids, for which new lightcurves have been observed during the past few years. The new data are summarized in Table 1 and lightcurves are shown in Fig. 2. The data are stored in SAPC (Standard Asteroid Photometric Catalog, http://www.astro.helsinki.fi/SAPC) which is the updated version of the Uppsala Asteroid Photometric Catalogue (UAPC, Lagerkvist et al., 2001). We also used previously published data that is stored in UAPC and SAPC. We considered all the lightcurves as relative photometry, since this is adequate for spin and shape determination, and the number of absolute observations was too small for studying phase-curve behaviour.

For (79) Eurynome, (130) Elektra, and (376) Geometria, we obtained an unambigu-

Figure 2 ous spin and shape solution, for (54) Alexandra, (93) Minerva, (471) Papagena, and (776) Berbericia a few possible spin solutions and, for (31) Euphrosyne, a constrained distribution of spin solutions. As mentioned above, errors of $5^{\circ}(\lambda)$ and $15^{\circ}(\beta)$ apply to each spin solution due to inaccuracies in the model shape and scattering law. The uncertainty of the period depends on the period itself and on the total time span of the observations, as explained in connection to $\mathrm{Eq}$ 1. The number of digits in the rotation periods corresponds to their accuracy in Table 2, where all the spin solutions are summarized. Some of the targets show double solutions, implying two solutions with the same rotation period and pole latitude, and pole longitudes roughly $180^{\circ}$ apart.

Table 2

The accuracy of spin solution is usually considered to depend on the number of apparitions but at least in this case, where the number of apparitions was five or more in every case, it did not correlate with the period accuracy. Neither did the total time span of the observations which was of the same order for all the targets. We, however, found a correlation between the number of lightcurves and the accuracy of the period solution. In general, the period 


\section{ACCEPTED MANUSCRIPT}

uncertainty for each spin solution is $\Delta P$ from Eq. 1. For all eight targets of this paper, $\Delta P$ for a single spin solution was about 0.00001 . However, for a majority of the targets, there were more than one spin solution, and thus the real period uncertainty was larger than $\Delta P$. The plot in Fig. 3 shows how the period uncertainty depends on the number of lightcurves. It seems that, when the number of lightcurves is around 10-13, the period uncertainty decreases sharply, then reaching more slowly the level of $\Delta P$, which is reached at around 30 lightcurves. This, of course, is highly sensitive to the quality of the lightcurves, but for a set of lightcurves covering close to one rotation, this kind of behaviour might be common in lightcurve inversion. A similar occurrence is present in asteroid orbit computation, where the uncertainty of the orbital parameters decreases sharply as the observational time interval increases to a certain point (e.g., Muinonen et al., 2006).

The number of lightcurves alone, however, is not a sufficient measure in the cases where the total time span of observations is short or the number of apparitions is low and we do not consider the case here. However, the uncertainty does not increase to infinity for limited data sets, but settles to a few percent of the rotation period for a single lightcurve covering one revolution. This is based on the period uncertainties obtained for targets with only a few lightcurves in Muinonen et al. (2007). It is left for future studies to find out if the sharp increase in period uncertainty is typical for lightcurve inversion, or was it just due to the relatively small number (eight) of sample objects.

For comparison to our results, there is a summary of previous solutions in the Database of asteroid spin vector determinations (http://vesta.astro.amu.edu.pl/Science/Asteroids/). The database was originally created by Per Magnusson, at that time working at the Uppsala Observatory (Sweden), and is currently maintained by Agnieszka Kryszczynska at the Poznan Observatory. Recent results of some targets included in this paper are found in Durech et al. (2007). Differences to the results in other publications are due to the use of different modelling techniques. Naturally, it enhances the reliability of the results if they are in 


\section{ACCEPTED MANUSCRIPT}

agreement with the ones obtained using other methods. On the other hand, if the results are different from others, further observations are needed to find the correct solution.

For the asteroid sizes, there are varying estimates in the literature. Our data for sizes and spectral classes have been collected from UAPC (Lagerkvist et al. 2001).

We assessed the need for weighting lightcurves according to the sampling density of lightcurve points (Eq. 2). However, adding weights had no effect to the solutions and, to be consistent with earlier solutions, we omitted them. This analysis showed again the stability of the convex inversion method. However, there may be cases, where the densely observed lightcurves dominate so strongly that they prevent finding a good fit for sparsely observed curves of high quality. In such cases, applying weights may stabilize the problem.

\section{(31) Euphrosyne, C, $247 \mathrm{~km}$}

We used ten lightcurves, one of which was new (Table 1). The other lightcurves were published in Kryszczynska et al. (1996), Barucci et al (1985), McCheyne et al. (1985), and Schober et al. (1980). The data spanned a total of 30 years. This is an example of a target with data sufficient to provide only a clearly restricted number of solutions instead of an unambiguous solution, although the amount of data is extensive (Fig. 4). The (P,rms)-plot shows that the best fits to the data are achieved with the period range of $P=5.5300-5.5340$ $\mathrm{h}$, but periods up to $5.5375 \mathrm{~h}$ are possible. The $(\lambda, \beta)$ plot shows that two distinct spin axis ranges, one around $\lambda=200^{\circ}, \beta=0^{\circ}$ and another one around $\lambda=330^{\circ}, \beta=-30^{\circ}$. Plotting $\lambda$ and $\beta$ against $r m s$ did not help to choose the correct pole and further observations should be planned according to the $(\lambda, \beta)$-plot to eliminate spurious solutions. In the Poznan database, there are also a wide range of solutions for this target none of which is, however, in agreement with our solution.

(54) Alexandra, C, $160 \times 135 \mathrm{~km}$

We used a data set of 20 lightcurves, two of which were not published before (Table 1). The other data were from Belskaya et al. (1993), Tancredi and Gallardo (1991), Di Martino 


\section{ACCEPTED MANUSCRIPT}

et al. (1987), Haupt and Hanslmeier (1985), and van Houten Groeneveld et al. (1979). The data spanned a total of 42 years. We obtained three possible solutions for the spin state, namely 1) $\lambda=307^{\circ}, \beta=20^{\circ}$, and $\left.P=7.02263 \mathrm{~h}, 2\right) \lambda=122^{\circ}, \beta=-36^{\circ}$, and $P=7.02334 \mathrm{~h}$, and 3) $\lambda=325^{\circ}, \beta=-37^{\circ}$, and $P=7.02334 \mathrm{~h}$, the latter two constituting a double solution. The shape solutions corresponding to the first and the double solution were different, and are not displayed here. There is a previous spin solution in the Poznan database, namely $\lambda=160 / 290^{\circ}, \beta=50^{\circ}$, and $P=7.026384 \mathrm{~h}$, which is different from ours

\section{(79) Eurynome, $S, 68 \mathrm{~km}$}

We used 33 lightcurves, one of which was not published before (Table 1). The other data were from Michalowski and Velichko (1990), Di Martino and Cacciatori (1984), De Angelis and Mottola (1995), Scaltriti and Zappalà (1976), Schober (1976), and Shevchenko et al. (1996). The total time span of the observations was 32 years. An unambiguous spin solution was obtained at $\lambda=64^{\circ}, \beta=15^{\circ}$, and $P=5.97857 \mathrm{~h}$. The shape solution is shown in Fig.

5. Our pole solution lies within ten degrees of one of the solutions in the Poznan database. Figure 5

(93) Minerva, C, $150 \mathrm{~km}$

14 lightcurves were used, five of which had not been published before (Table 1). The rest of the data are published in Millis et al. (1985), Debehogne et al. (1982), and Denchev (2000, in Lagerkvist et al. 2001). The total time span of the observations was 26 years. Two solutions were accepted, namely 1) $\lambda=49^{\circ}, \beta=-40^{\circ}$, and $P=5.98313 \mathrm{~h}$ and 2) $\lambda=216^{\circ}, \beta=21^{\circ}$, and $P=5.98088 \mathrm{~h}$. The shape solutions for both pole directions were similar, and the model is shown in Fig. 6. The previous spin solution in the Poznan database is $\lambda=196^{\circ}, \beta=13^{\circ}$, and $P=5.9784 \mathrm{~h}$.

(130) Elektra, G, $215 \times 155 \mathrm{~km}$

We used 58 lightcurves of Elektra, four of which were new (Table 1), and the rest are published in Danforth and Ratcliff (1994), Debehogne et al. (1990), Harris and Young 


\section{ACCEPTED MANUSCRIPT}

(1989), Weidenschilling et al. (1987), Shevchenko et al. (1996), and Durech et al. (2007). The total time range of the observations was 26 years. The solution that fitted the data the best was $\lambda=160^{\circ}, \beta=-85^{\circ}$, and $P=5.22466 \mathrm{~h}$. The corresponding shape model is shown in Fig. 7. The solution is the same, within error limits, as the one by Durech et al. (2007) $\left(\lambda=65^{\circ}, \beta=-88^{\circ}\right.$, and $P=5.224664 \mathrm{~h}$.

(376) Geometria, $S, 36 \mathrm{~km}$

We used 35 lightcurves of Geometria, one of which was new. The old data were published in Kryszczynska et al. (1996), Barucci and Di Martino (1984), Hainaut-Rouelle et al. (1995), Zeigler (1987), and Michalowski et al. (2005). Observations ranged a total of 21 years. The best fit solution was $\lambda=68^{\circ}, \beta=2^{\circ}$, and $P=7.71002 \mathrm{~h}$. The shape model is shown in Fig.

8. The pole longitude is similar to earlier solutions, but the latitude is different.

\section{(471) Papagena, S, $140 \mathrm{~km}$}

We used 13 lightcurves, one of which was new (Table 1). The older data has been published in Lustig (1977), Di Martino and Cacciatori (1984), Scaltriti and Zappalà (1978), and Surdej and Surdej (1977). The total time span of the observations was 31 years. We obtained three acceptable spin solutions: 1) $\lambda=29^{\circ}, \beta=41^{\circ}$, and $P=7.7 .11364 \mathrm{~h}, 2$ ) $\lambda=222^{\circ}, \beta=40^{\circ}$, and $P=7.11246 \mathrm{~h}$, and 3$) \lambda=236^{\circ}, \beta=56^{\circ}$, and $P=7.11512 \mathrm{~h}$. The corresponding shape solutions are different, and are not displayed here. The first of the pole solutions is close to one of the two earlier solutions in the Poznan database.

(776) Berbericia, C, $390 \mathrm{~km}$

33 lightcurves were used. Two of the curves were unpublished (see Table 1), and the rest published in Debehogne et al. (1983), Di Martino et al. (1987), Schober (1979), HainautRouelle et al. (1995), and Durech et al. (2007). The observations spanned a total of 28 years. Two solutions that fitted the data equally well were 1) $\lambda=170^{\circ}, \beta=+59^{\circ}$, and $P=7.66701 \mathrm{~h}$ and 2) $\lambda=347^{\circ}, \beta=+11^{\circ}$, and $P=7.66701 \mathrm{~h}$. The shape solutions were 
otherwise similar, but mirror images through horizontal reflection. The shape corresponding to the solution $\lambda=170^{\circ}, \beta=+59^{\circ}$ is shown in Fig. 9. Durech et al. (2007) obtained a single solution $\lambda=347^{\circ}, \beta=+12^{\circ}$, and $P=7.66701 \mathrm{~h}$, in agreement with one of our solutions.

Figure 9

\subsection{Characterization of shape solutions}

We used a set of 87 convex inversion shape solutions, five of which are published in this paper, and the rest in K01, Kaasalainen et al. (2002a,b; 2003, 2004), Slivan et al. (2003), T03, Michalowski et al. (2004, 2005, 2006), Marchis et al. (2006), and Durech et al. (2007). Also the Database of Asteroid Models from Inversion Techniques (http://astro.troja.mff.cuni.cz/projects/asteroi was used in collecting the data. In the following, we discuss the ranges of axis ratios as well as correlations between shape, size, rotation period, and taxonomic type. It has to be kept in mind that, especially for non-elongated objects, the vertical dimension of the model shapes is somewhat ill-defined when using only relative photometric observations. Otherwise, the features and dimensions of the shapes have been shown to be stable, e.g., against using various scattering laws in lightcurve inversion (K01). Taxonomic types are defined according to the SMASS classification (Bus and Binzel 2002). Most of the asteroids included in this study belong to the three main categories $\mathrm{C}, \mathrm{S}$, and $\mathrm{X}$, and two are determined as D-type. Properties of all the sample asteroids are collected in Table 4.

\subsubsection{Axis ratios}

If we define the overall dimensions and axis lengths of the characteristic ellipsoids as $c<b<$ $a$, the corresponding principal moments are $C_{c}>C_{b}>C_{a}$. To make comparison of these quantities easier, we discuss the axis ratios that lie in the range $[0,1]$; in the former case this corresponds to $c / a, c / b$ and $b / a$, while in the latter case to $C_{a} / C_{c}, C_{b} / C_{c}$, and $C_{a} / C_{b}$. For the OD and E1 techniques, the definition of the $c$-axis is straightforward, since it is fixed to be aligned with the rotation axis. For the other two methods, where all the axes are allowed 
to vary, we define the $c$-axis as the one closest to the original rotation axis.

In many other contexts, shapes are characterized with elongation $(1-b / a)$ and flatness $(1-c / b)$ but, in asteroid research, axis ratios have been in common use. Thus, an elongated asteroid has a low $b / a$-ratio, while a flat asteroid has a low $c / b$-ratio.

Table 3

In Table 3, we show the mean, standard deviation, and maximum and minimum values for the axis ratios obtained with the four different methods. In general, the methods give similar mean values, except for the $C_{b} / C_{c}$-ratio of PMI, whose mean is lower than the other corresponding ratios. The value of $b / a$ is, by definition, constrained to the range $[0,1]$ for all the methods, but $c / b$ is allowed to exceed unity for the OD and PMI techniques. In Figs. $10 \mathrm{a}$ and $\mathrm{b}$, we see that the first three approaches (OD, E1, and E2) give similar results for individual targets, whereas the results of the PMI technique are clearly different (Fig. 10c). Thus, the axis ratios from the first three methods can be compared with one another, while the principal moments should be considered separately.

Figure

We use the first three methods to define the actual dimensions of the asteroids. In Fig. 10 11a we show the distributions of dimensional ratios $(c / b, b / a)$ from the E2 technique (the OD and E1 techniques produce similar distributions). We see, that $c / b$ and $b / a$ most commonly lie within the range $[0.7,1], b / a$ getting slightly lower values. There are a few objects that are significantly elongated with $b / a$ below 0.6 . These can be binary asteroids, or Eros and Itokawa -like elongated objects. The $c / a$-ratio usually lies within the range $[0.5,1]$. The results are in accordance with laboratory and numerical simulations (see Holsapple et al. 2002), which suggest that, for fractured shapes, $b / a=[0.6,0.7]$ and $c / a=[0.45,0.5]$.

Figure

There is an interesting feature of S- and D-type objects achieving significantly smaller 11 $b / a$ ratios than C- and X-types. From Bus and Binzel (2002), it is evident, that C- and X-type asteroids are spectrally close to one another, while the spectra of S- and D-types deviate from each other and from those of the C- and X-types. The S-types also have higher albedos than the $\mathrm{C}$ - and $\mathrm{X}$-types. The C-types are known to be of lower density than the 


\section{ACCEPTED MANUSCRIPT}

other spectral types, which could explain the more spherical shapes, since the energy of a collision, strong enough to fracture, e.g., an S-type asteroid, is absorbed into the interior by breaking the structure of the porous material inside a C-type asteroid, thus producing a global crater on the surface of the target (e.g., asteroid (253) Mathilde), but leaving it otherwise unfractured. Further studies with more observations of different spectral types are called for to shed light on this possible correlation between the spectral types and shape.

\subsubsection{Shape, rotation period, and size}

Having at hand methods to characterize the shapes of convex bodies, we can study the correlations between shape and other physical properties. In the following, we show distributions with dimensions obtained using only the E2 technique, since other characterization techniques give similar results.

There is dependence between shape and rotational period as seen in Fig. 12, since all of the most elongated objects have periods between $4.5-10 \mathrm{~h}$, while the ones with axial ratios $b / a>0.7$ are slow or fast rotators. It is also interesting, that only C- and S-type asteroids have periods over $11 \mathrm{~h}$. There is no evident explanation for these results, and more data are required to make any further conclusions. Especially, long-period targets $(P>15 \mathrm{~h})$ lack proper lightcurve data. Also, data of short-period asteroids $(P<2 \mathrm{~h})$ would be of interest since, for these, the centrifugal forces begin to override the internal forces, and loose rubble pile asteroids may begin to separate into parts. This should be seen in the elongation of these objects.

The shape vs size plot (Fig. 13) also shows non-random features: as would be expected, 12 the small objects reach more elongated shapes than the large ones while spherical shapes are represented by the entire size range. Observations of the largest asteroids are called for to provide information about the limit above which the asteroids are spherical. This limit should also depend on the taxonomic type. In our sample, the largest asteroid, (2) 


\section{ACCEPTED MANUSCRIPT}

Pallas $(D=523 \mathrm{~km})$, is spherical, but the second largest, $(10)$ Hygiea $(D=429 \mathrm{~km})$, is clearly elongated with $b / a=0.75$. Both are C-type asteroids. Taxonomic and shape data of asteroids smaller than $30 \mathrm{~km}$ in diameter is now almost completely lacking, and thus more observations of this population are also called for.

Figure

\subsubsection{Principal moments}

The principal moments of the shape models are not suitable for the determination of the axis ratios, since they give systematically lower values than the techniques giving the actual dimensions (Fig 10c). They can, however, be used to estimate the goodness of the convex shape model.

The axis of the largest inertia of the convex shape model does not usually coincide with the rotation axis of the model. One plausible explanation for this is that the lightcurve features caused by non-convex shape features and possible albedo markings in the target asteroids are reproduced by the convex model shapes with homogenous albedo distribution, distorting the shape somewhat, and causing deviation from principal-axis rotation. However, even slight changes in shape of nearly spherical models may change the directions of the principal axis significantly. Principal axes are also unstable for models with $b / a$-ratio close to unity since the vertical dimension for such objects is ill-defined. Thus, knowing the angle $\theta$ between the axis of largest inertia and axis of rotation of the model shape may provide us information about the scale of non-convexities or albedo features of target shapes with $b / a$-ratio significantly different from unity.

As seen in Fig. 14, $\theta$ reaches values up to $45^{\circ}$. For elongated objects it seems to be smaller, increasing with increasing $b / a$-ratio. A large range of $\theta$ values would be expected for objects with $b / a>0.9$ but, curiously, it decreases sharply with $b / a$ increasing from 0.9 to 1. Along with increasing $c / b$-ratio, the deviation increases steadily towards spherical shapes. As an example, elongated shapes with $b / a$-ratio below 0.7 usually have $\theta<20^{\circ}$. A few such 
objects, however, show larger $\theta$ values, suggesting strongly non-convex shape, or significant variegation of albedo on the surface.

Figure

In Fig. 11d, we see that two of the shape models have principal moment ratios $C_{b} / C_{c} \quad 14$ above unity, which implies an unstable rotation state. Such solutions exist, since the shapes have been inspected only by eye, and it is impossible to decide if an irregular object has $C_{b} / C_{c}=1$ or a bit larger. Slight excesses can be tolerated for two reasons. First, the dimension along the spin axis of the shape solution is sensitive to the scattering law. For determining the shape solutions used in this paper, a combination of Lommel-Seeliger and Lambert scattering laws has been used, and the weight of the Lambertian part has been about $10 \%$. However, this scattering law is inaccurate for expressing the scattering behaviour of asteroid surfaces, and we do not know what the optimal weight for each asteroid would exactly be. Without altering the shape otherwise, the vertical dimension of the model can be moderately altered by changing the weight of the Lambertian part.

If the vertical stretching in principal-moments sense was only due to the ill-defined scattering law, there would be basis for changing the scattering parameters to produce a more spherical shape. However, another reason for too large $C_{b} / C_{c}$-ratios is the same as that for the tilt angle of axis of largest inertia to the rotation axis of the model - the convexity of the shape model. For these reasons we should be careful when considering refinement of the vertical axis dimension of the shape solution. This dimension is anyway the least constrained quantity of the shape model, when only relative photometry is available. If the vertical stretching is small, not visible to an eye, the solution can be accepted.

\subsubsection{Irregularity of shapes}

One way to demonstrate the irregularity of a shape is to compute the deviation from the best-fit ellipsoid. Here we use the root-mean-square value $(\mathrm{rms})$ of the ellipsoid fit from the E2 technique 


$$
r m s=\sqrt{\frac{\chi_{e}^{2}}{n_{v}}},
$$

where $\chi_{e}^{2}$ is obtained from Eq. 7, and $n_{v}$ is the number of vertices. As seen in Fig. 15, rms lies within range $[0.02,0.06]$. Curiously, all the most elongated objects with $b / a<0.65$ have $r m s<0.04$. There are only a few such objects, however, and more observations are needed to see if this is a real phenomenon. Towards flatter shapes, i.e., along with decreasing $c / b$-ratio, the shapes seem to become more irregular in general. Otherwise, there does not seem to be a correlation between rms and elongation or flatness. Between size and rms, there is a slight negative correlation with Pearson product-moment correlation coefficient $r=-0.32$. This seems natural, since large objects can be expected to be more regular in shape than small ones. Between rms and taxonomic type, there is no correlation.

The targets with small rms have lightcurves characteristic for ellipsoidal shapes, i.e., 15 two equal minima and maxima per lightcurve period, and they might as well be modelled as ellipsoids. Most asteroids, however, have lightcurves with more than two minima and maxima or unequal maxima or minima. If such objects are modelled with an ellipsoid, a lot of information is lost.

Another way do describe the irregularity of a polyhedron is to calculate how large a portion of the model surface is covered with large planar areas. When this portion increases, the irregularity of the objects increases as well. Since the distribution of the facet areas cannot be guaranteed to be independent on the shape model, using the distribution of facet areas as a classification method requires that all the models are obtained with the same modelling technique and the same number of shape parameters. In Table 4, we show, as an example, quantities $f_{1}$, the fraction of the facets with area more than $20 \%$ of the largest facet area, and $f_{2}$, the fraction of the largest facet area of the total surface area (we give these quantities for only 52 shapes, since the exact modelling technique for the rest was 
unclear. $f_{1}$ and $f_{2}$ are somewhat correlated with Pearson's $r=-0.57$, i.e., when the number of large facets decreases, the fraction of the total surface area covered by the largest facet increases. In Fig. 16, $f_{1}$ is plotted against the E1 ellipsoid axis ratios. We see that, Figure towards more spherical shapes, the number of large facets increases, which means a more 16 even distribution of facet sizes, and implies a more regular shape. Results are in agreement with the observation that, on elongated shapes, only a few facets often comprise a large fraction of the total surface area. According to simulations and test analysis, these large facets can be interpreted to show potential locations of global-scale concavities (KT01, K01). Within our sample, only C-type asteroids have $f_{1}>0.12$, while the most dense cluster of $\mathrm{X}$ types is located in $f_{1}<0.05$. This implies that, among the C-type asteroids, there are more regular bodies than among other spectral types. This is in agreement with the assumption that the C-type asteroids, consisting of more porous material than the other types, are also less subjected to fractionation than other types. In Fig. 17 we show how the facet sizes are distributed in decreasing order on the surfaces of three different model shapes. The model of (776) Berbericia in Fig. 17c shows that a large part of the surface area is covered by only a few facets, while the model of (2) Pallas (Fig. 17a) has more evenly distributed facet areas. This implies that Berbericia is irregular, while Pallas has more soft features. The case of (6) Hebe lies in between these. The linear-exponential shape of the distribution is typical for models for which facets were combined for Minkowski minimization after the convex inversion procedure. The sharp decrease of size in the small-end of the facets distribution is caused by the combination procedure, which leaves some facets unchanged. In Table 4, we give the slope $k$ of the linear part for the 34 models, and in Fig. 18 we plot $k$ against size. Decreasing value of $k$ means more evenly distributed facet areas, and thus, a more regular Figure shape. According to the plot, the C-type asteroids get more irregular with decreasing size, 18 while other types show more shattered $k$ values.

In Fig. 19 we see that, as the dominance of the largest facets increases, the deviation $\theta$ 
of the axis of the largest moment of inertia from the rotation axis increases as well. Objects with both large $\theta$ and small $f_{1}$ are likely to contain global scale nonconvexities, locations of which might be incicated by the locations of the largest facets.

Figure

We also calculated the shapes of the largest facets as the axis ratios of the best-fit ellipse. 19 There was no evident correlation with facet shape and model shape or taxonomic type. Thus, facet shapes cannot be used to define the existence of global scale circular craters, expected to exist mainly on C-type asteroids.

\section{Conclusions and future work}

Three approaches are presented for calculating the global dimensions of convex polyhedron shape models of asteroids, and the results from all the techniques are in agreement. The relatively good ellipsoid fits to model shapes suggest that the surface irregularity of asteroids might be described by multiplying the best-fit ellipsoid by a spherical harmonics series, as was done for potato tubers by Torppa et al. (2006). This would be a continuation to the work of Muinonen and Lagerros (1998), who modelled asteroids statistically with Gaussian spheres. And, after all, asteroid and potato tuber shapes are not that far from each other.

The principal moments of inertia may be used to describe the dynamical state of the model, providing information about how well it describes the shape of the target asteroid. Notable deviation of the principal axis of the model shapes from their rotation axis, especially for elongated objects and along with the existence of large planar areas, may reflect presence of large-scale concavities or strong albedo variegation on the surface. To evaluate the reliability of this interpretation, however, we have to carry out a large number of simulations for various shapes and scattering properties.

In addition to the OD, E1, E2, and PMI techniques, we calculated the principal components, i.e., the Karhunen-Loeve transform, for each model shape by sampling evenly a grid 
of points on their surfaces. Axis ratios, however, differed significantly from those of the other methods, and it is left for future studies to find a physical interpretation for these quantities, and possibly a more suitable way to sample the points within the shape.

The statistical study of various physical properties of asteroids shows interesting features: S-type asteroids achieve smaller $b / a$ ratios than C- and X-types, large asteroids, as well as those with long rotation periods have less elongated shapes, and C-type objects dominate the population of large asteroids. Original facet information from the convex inversion procedure should be preserved since, it seems, that the distribution of facet areas is in somewhat binded to other physical properties and thus, could be used to classify asteroids. Combining facets for Minkowski minimization seems to change the original facet area distribution, and produce a logarithmic distribution for the intermediate size range, while preserving the spike for the largest facets (Fig. 17), showing those shapes in which large planar areas exist. Additional shape data are required, however, to make further conclusions about classifying asteroids according to the slope of the linear part of the logarithmic distribution. Additional shape, size, and taxonomic data of the extreme populations (those with diameters $D<30 \mathrm{~km}$ and $D>300 \mathrm{~km}$, and with periods $P<2 \mathrm{~h}$ and $P>15 \mathrm{~h}$ ) are, however, called for in order to perform a more thorough analysis of the asteroid population. Also, different orbital populations should be separated in statistical studies, but also this requires a lot more observational data. Especially, radar-derived shapes would increase the number of small, kilometer-sized near-Earth asteroids available for characterization. The shape solutions derived from the radar observations or spacecraft flybys, however, cannot be included in the analysis at the moment, since none of our techniques allows processing of non-star-like shapes. Thus, we plan to develop the existing techniques and, in the future, include also non-star-like shapes in the analyses. 


\section{Acknowledgments}

The study has been funded by Vilho, Yrjö and Kalle Väisälä foundation, the Academy of Finland, the Finnish Graduate School in Astronomy and Space Physics, and the University of Helsinki. JT thanks all the Finnish amateur astronomers who took part in carrying out the observations.

\section{References}

Belskaya I.N., Dovgopol A.N., Erikson A., Lagerkvist C-I., and Oja T., 1993. Physical studies of asteroids XXVII: Photoelectric photometry of asteroids 14 Irene, 54 Alexandra and 56 Melete, A\&A SS., 101, 507-511.

Barucci M.A. and Di Martino M., 1984. Rotational rates of very small asteroids - 124 Brunhild, 376 Geometria, 437 Rhodia, and 1224 Fantasia. A\&A SS., 57, 103.

Barucci M.A., Fulchignoni M., Burchi R., and D'Ambrosio V., 1985. Rotational properties of ten main belt asteroids - Analysis of the results obtained by photoelectric photometry, Icarus, 61, 152 .

Bus S. and Binzel R., 2002. Phase II of the small main-belt asteroid spectroscopic survey. A feature based taxonomy, Icarus, 158, 146.

Danforth C.W. and Ratcliff S.J., 1994. CCD Photometry of 130 Elektra. MPB, 21, 1-3.

De Angelis G. and Mottola S., 1995. Lightcurves and pole determinations for the asteroids 69 Hesperia, 79 Eurynome and 852 Wladilena, Planet. Space Sci. 43, 1013.

Debehogne H., Lagerkvist C-I., and Zappalà V., 1982. Physical studies of asteroids VIII Photoelectric photometry of asteroids 42, 48, 93, 105, 145, and 245, A\&A SS., 50, 277.

Debehogne H., De Sanctis G., and Zappalà V., 1983. Photoelectric photometry of asteroids 45, 120, 776, 804, 814, and 1982DV, Icarus, 55, 236. 


\section{ACCEPTED MANUSCRIPT}

Debehogne H., Lagerkvist C.-I., Magnusson P., and Hahn G., 1990. Physical studies of asteroids XX - Photoelectric photometry of asteroids, in Asteroid, Comets and Meteors III. eds. C.-I. Lagerkvist, H. Rickman, B.A. Lindblad, and M. Lindgren. Uppsala Universitet, Reprocentralen, HSC, Uppsala, p 45.

Demura H., Kobayashi S., Nemoto E., and 17 co-authors, 2006. Pole and global shape of 25143 Itokawa, Science 312, 1330.

Di Martino M. and Cacciatori S., 1984. Photoelectric photometry of 14 asteroids, Icarus, 60,75 .

Di Martino M., Zappalà V., De Sanctis G., and Cacciatori S., 1987. Photometric photometry of 17 asteroids. Icarus 69, 338.

Drummond J. D., Weidenschilling S. J., Chapman C. R., and Davis D. R., 1991.

Photometric geodesy of main-belt asteroids. IV - an updated analysis of lightcurves for poles, periods, and shapes, Icarus, 89, 44.

Dunham D.W., 2006. Upcoming Asteroid Occultations, Sky \& Telescope, June 2006, p. 63.

Durech J., Kaasalainen M., Marciniak A., and 40 co-authors, 2007. Physical models of ten asteroids from an observers' collaboration network, A\&A 465, 331.

Hainaut-Rouelle M.-C., Hainaut O.R., and Detal A., 1995. Lightcurves of selected minor planets. A\&A SS., 112, 125-142.

Harris A.W. and Young J.W., 1989. Asteroid lightcurve observations from 1979-1981, Icarus, 81, 314.

Hartmann W. K., Binzel R. P., Tholen D. J., Cruikshank D. P., and Goguen J., 1988. Trojan and Hilda asteroid lightcurves. I - Anomalously elongated shapes among Trojans (and Hildas?), Icarus, 73, 487.

Haupt H. and Hanslmeier A., 1985. in Mitt.der Univ. Sternwarte Graz 107. 
Holsapple K., Giblin I., Housen K., Nakamura A., and Ryan E., 2002. Asteroid Impacts: Laboratory Experiments and Scaling Laws. In Asteroids III ( W. Bottke, A. Cellino, P. Paolicchi, and R.P. Binzel, Eds.) p. 443, Univ. of Arizona press, Tuscon. van Houten-Groeneveld I., van Houten C.J., and Zappalà V., 1979. Photoelectric photometry of seven asteroids, A\&A SS., 35, 223.

Kaasalainen M., Lamberg L., Lumme K., and Bowell E., 1992. Interpretation of lightcurves of atmosphere less bodies. I. General theory and new inversion schemes, A\&A 259, 318-332. Kaasalainen M. and Torppa J., 2001. Optimization methods for asteroid lightcurve inversion. I. Shape determination, Icarus, 153, 24-36.

Kaasalainen M., Torppa J. and Muinonen K., 2001. Optimization methods for asteroid lightcurve inversion. II. The complete inverse problem, Icarus, 153, 37-51.

Kaasalainen M., Torppa J., and Piironen J., 2002a. Models of twenty asteroids from photometric data, Icarus, 159, 369-395.

Kaasalainen M., Torppa J., Piironen J., 2002b. Binary structures among large asteroids. A\&A. 383, 19 .

Kaasalainen M., Kwiatkowski T., Abe M., and 14 co-authors, 2003. CCD Photometry and model of MUSES-C target (25143) 1998 SF36. Astron. Astrophys. 405, L29.

Kaasalainen M., Pravec P., Krugly Yu. N., and 19 co-authors, 2004. Photometry and models of eight near-Earth asteroids. Icarus 167, 178.

Kryszczynska A., Colas F., Berthier J., Michalowski T., and Pych W., 1996. CCD Photometry of Seven Asteroids: New Spin Axis and Shape Determinations, Icarus 124, 134 Lagerkvist C-I., Piironen J., and Erikson A., 2001. Asteroid Photometric Catalogue, $5^{\text {th }}$ update, Uppsala University Press, Uppsala. 


\section{ACCEPTED MANUSCRIPT}

Liu Y., Schnare D., Park J.S., Hill E., Eimer B., and Taylor L.A., 2007. Shape analysis of lunar dust particles for astronaut toxicological studies. Proceedings of the 38th Lunar and Planetary Science Conference, p. 1383.

Lustig G., 1977. The period of rotation and the photoelectric lightcurve of the minor planet 471 Papagena, A\&A SS., 30, 117.

Magnusson P., 1990. Spin vectors of 22 large asteroids, Icarus, 85, 229.

Magri C., Ostro S., Scheeres D., Nolan M., Giorgini J., Benner L., Margot J-L., 2007. Radar observations and a physical model of Asteroid 1580 Betulia, Icarus, 186, 152.

Marchis F., Kaasalainen M., Hom E.F.Y., Berthier J., Enriquez J., Hestroffer D., Le Mignant D., and de Pater I., 2006. Shape, size and multiplicity of main-belt asteroids, Icarus, 185,39 .

Maria A. and Carey S., 2002. Using fractal analysis to quantitatively characterize the shapes of volcanic particles, J. of Geophys. Res., 107.

McCheyne R.S., Eaton N., and Meadows A.J., 1985. Visible and near-infrared lightcurves of eight asteroids, Icarus, 61, 443.

Michalowski T. and Velichko F.P., 1990. Photoelectric photometry, parameters of rotation and shapes of asteroids 22 Kalliope and 79 Eurynome, Acta Astron., 40, 321.

Michalowski T., Kwiatkowski T., Kaasalainen M., and 8 co-authors, 2004. Photometry and models of selected main-belt asteroids (I): 52 Europa, 115 Thyra, and 382 Dodona. A\&A, $416,353$.

Michalowski T., Kaasalainen M., Marciniak A., and 8 co-authors, 2005. Photometry and models of selected main-belt asteroids (II): 173 Ino, 376 Geometria, and 451 Patientia. Astron. Astrophys. 443, 329. 


\section{ACCEPTED MANUSCRIPT}

Michalowski T., Kaasalainen M., Polińska M., Marciniak A., Kwiatkowski T., Kryszczynska A., and Velichko F. P., 2006. Photometry and models of selected main-belt asteroids (III): 283 Emma, 665 Sabine, and 690 Wratislavia. Astron. Astrophys. 459, 663.

Millis R.L., Wasserman L.H., Bowell E., Franz O.G., Nye R., Osborn W., and Klemola A., 1985. The occultation of $\mathrm{AG}+29^{\circ} 398$ by 93 Minerva, Icarus, 61,124 .

Muinonen K., 1998. Introducing the Gaussian shape hypothesis for asteroids and comets, A\&A, 332, 1087.

Muinonen K., 2006. Inversion of small-particle silhouettes for Gaussian-sphere parameters, 9th Conference on Electromagnetic and Light Scattering by Nonspherical Particles: Theory, Measurements, and Applications, Book of Abstracts, N. Voshchinnikov, Ed., p. 203.

Muinonen K. and Lagerros J.S.V., 1998. Inversion of shape statistics for small solar system bodies, A\&A, 333, 753 .

Muinonen K., Virtanen J., Granvik M., and Laakso T., 2006. Asteroid orbits using phase-space volumes of variation, MNRAS, 368, 809.

Muinonen K., Torppa J., Virtanen J., Näränen J., Niemelä J., Granvik M., Laakso T., Parviainen H., Aksnes K., Dai Z., Lagerkvist C.-I., Rickman H., Karlsson O., Hahn G., Michelsen R., Grav, T., Pravec P., and Jørgensen U. G., 2007. Spins, shapes, and orbits for potentially hazardous near-Earth objects by NEON. In Proceedings of IAU Symposium No 236, Near-Earth Objects, our Celestial Neighbors: Opportunity and Risk (A. Milani, G.

Valsecchi, and D. Vokrouhlicky, eds.).

Press W. H., Flannery B.P., Teukolsky S.A., and Vetterling W.T., 1994. Numerical Recipes in Fortran 77, Cambridge University Press, Cambridge, UK.

Scaltriti F. and Zappalà V., 1976. A photometric study of the minor planets 192 Nausikaa and 79 Eurynome, A\&A SS., 23, 167. 


\section{ACCEPTED MANUSCRIPT}

Scaltriti F. and Zappalà V., 1978. Photoelectric photometry of asteroids 37, 80, 97, 216, 270, 313, and 471, Icarus, 34, 428.

Schober H.J., 1976. The period of rotation and the photoelectric lightcurve of the minor planet 79 Eurynome, Icarus, 28, 415.

Schober, H.J., 1979. 87 Aquitania and 776 Berbericia - Two slow spinning asteroids with rotation periods of nearly one day. A\&A SS., 38, 91.

Schober H.J., Scaltriti F., Zappalà V., and Harris A.W., 1980. The remaining large minor planets with unknown rotational properties - 31 Euphrosyne and 65 Cybele, A\&A, 91, 1.

Shevchenko V.G., Chiorny V.G., Kalashnikov A.V., Krugly Yu.N., Mohamed R.A., and Velichko F.P., 1996. Magnitude-phase dependencies for three asteroids, A\&A SS., 115, 475-479.

Slivan S., Binzel R. P., Crespo S., Lucy D., Kaasalainen M., Lyndaker M. M., Krco M., 2003. Spin vectors in the Koronis family: Comprehensive results from two independent analyzers of 213 rotation lightcurves. Icarus 162, 285.

Surdej A. and Surdej J., 1977. Rotation period and photoelectric lightcurves of Asteroid 471 Papagena, A\&A SS., 30, 121.

Tancredi G. and Gallardo T., 1991. A comparison of two pole determination methods for asteroids, A\&A 242, 279.

Tonon F., 2004. Explicit exact formulas for the 3-D tetrahedron inertia tensor in terms of its vertex coordinates, Journal of Mathematics and Statistics, 1(1),8-11.

Torppa J., Kaasalainen M., Michalowski T., Kwiatkowski T., Kryszczyńska A., Denchev P. and Kowalski R., 2003. Shapes and rotational properties of thirty asteroids from photometric data, Icarus 164, 346-383. 
Torppa, J., Valkonen, J. P. T., Muinonen, K., 2006. Three-dimensional stochastic shape modelling for potato tubers. Potato Res., 49, 109.

Weidenschilling S.J., Chapman C.R. Davis D.R., Greenberg R., Levy D.H., and Vail S., 1987. Photometric geodesy of main-belt asteroids. I - Lightcurves of 26 large, rapid rotators, Icarus 70, 191.

Zeigler K.W., 1987. Photoelectric photometry of asteroids 213 Lilaea and 376 Geometria. MPBu, 14, 11. 


\section{tables}

Table 1. Information about the photometric lightcurve observations published in this study. Columns contain the following information: 1) asteroid name, 2) date of observation, 3) phase angle, and 4) observatory code (TH-Taurus Hill, SR-Slope Rock, JK-Jakokoski).

\begin{tabular}{llll}
\hline Asteroid & Obs date & $\alpha\left(^{\circ}\right)$ & Observatory \\
\hline (31) Euphrosyne & Mar 30, 2007 & 21.7 & TH \\
(54) Alexandra & Dec 15, 2006 & 11.8 & SR \\
& Jan 21, 2007 & 18.5 & TH \\
(79) Eurynome & Feb 4, 2006 & 21.9 & TH \\
(93) Minerva & Dec 22, 2005 & 8.5 & JK \\
& Jan 14, 2006 & 14.5 & JK \\
& Dec 24, 2006 & 16.0 & TH \\
& Jan 30, 2007 & 5.9 & TH \\
& Feb 21, 2007 & 4.4 & SR \\
(130) Elektra & Feb 25, 2005 & 4.1 & JK \\
& Mar 1, 2005 & 5.5 & JK \\
& Mar 18, 2005 & 10.4 & JK \\
& Apr 22, 2006 & 7.8 & TH \\
(376) Geometria & Mar 26, 2006 & 21.5 & TH \\
(471) Papagena & Mar 25, 2007 & 16.9 & TH \\
(776) Berbericia & Mar 27, 2005 & 7.9 & JK \\
& Apr 1, 2005 & 8.5 & JK
\end{tabular}




\section{ACCEPTED MANUSCRIPT}

Table 2. Summary of the spin solutions. Columns are as follows: 1) asteroid name, 2) pole direction as ecliptic longitude and latitude, 3) rotation period, 4) rms-residual of the fit, 5) number of lightcurves, 6) number of apparitions, and 7) total time span of the observations.

\begin{tabular}{lllllll}
\hline Asteroid & $\lambda, \beta\left(^{\circ}\right)$ & $P(h)$ & $\mathrm{rms}$ & $N_{\text {cur }}$ & $N_{\text {app }}$ & $\mathrm{T}(\mathrm{yr})$ \\
\hline (31) Euphrosyne & distribution & $5.534 \pm 0.004$ & $0.012-$ & 10 & 5 & 30 \\
& & & 0.0125 & & & \\
(54) Alexandra & 307,20 & 7.02263 & 0.016 & 20 & 5 & 42 \\
& $122,-36$ & 7.02334 & 0.017 & & & \\
& $325,-37$ & 7.02334 & 0.017 & & & \\
(79) Eurynome & 64,15 & 5.97857 & 0.014 & 33 & 5 & 32 \\
(93) Minerva & $49,-40$ & 5.98313 & 0.015 & 14 & 5 & 26 \\
& 216,21 & 5.98088 & 0.014 & & & \\
(130) Elektra & $160,-85$ & 5.22466 & 0.019 & 58 & 12 & 26 \\
(376) Geometria & 68,2 & 7.71002 & 0.021 & 35 & 10 & 21 \\
(471) Papagena & 29,41 & 7.11364 & 0.021 & 13 & 3 & 31 \\
& 222,40 & 7.11246 & 0.021 & & & \\
(776) Berbericia & 170,59 & 7.66701 & 0.014 & 33 & 7 & 28
\end{tabular}




\section{ACCEPTED MANUSCRIPT}

Table 3. Comparison of the results of shape characterization using the four different methods. Colums contain: 1) method, 2) axial ratio, 3) mean value of the axial ratio, 4) standard deviation of the axial ratios, 5-6) maximum and minimium values of the axial ratios.

\begin{tabular}{llllll}
\hline method & ratio & $\sigma$ & mean & $\max$ & $\min$ \\
\hline Overall dimensions & $b / a$ & 0.1119 & 0.8463 & 0.9989 & 0.4903 \\
& $c / b$ & 0.1258 & 0.8709 & 1.2102 & 0.5203 \\
Ellipsoid fit (facets) & $b / a$ & 0.1061 & 0.8478 & 1.0000 & 0.5556 \\
& $c / b$ & 0.1131 & 0.8790 & 1.0000 & 0.4762 \\
Ellipsoid fit (radius) & $b / a$ & 0.1232 & 0.8348 & 0.9945 & 0.4343 \\
& $c / b$ & 0.0942 & 0.8670 & 1.0000 & 0.6550 \\
Principal moments & $C_{a} / C_{b}$ & 0.1191 & 0.8588 & 0.9977 & 0.4978 \\
& $C_{b} / C_{c}$ & 0.1320 & 0.8124 & 1.3494 & 0.4788
\end{tabular}


Table 4. List of asteroids included in the statistical analysis. Colums contain: 1) asteroid name, 2) $b / a, 3) c / b, 4)$ rotation period, 5) diameter (from the IRAS minor planet survey), 6) taxonomic type, 7) rms of the E2 ellipsoid fit, 8) fraction of the total surface area covered by the largest facet, 9) amount of facets with area $20 \%$ of the area of the largest facet, and 10) slope of the facet size distribution.

\begin{tabular}{|c|c|c|c|c|c|c|c|c|c|}
\hline Asteroid & $b / a$ & $c / b$ & $P(h)$ & $D(k m)$ & Tax & $r m s_{e}$ & $f_{1}$ & $f_{2}$ & $k$ \\
\hline (2) Pallas & 0.95 & 0.97 & 7.813225 & 523 & $\mathrm{C}$ & 0.026 & 0.020 & 0.278 & -0.0046 \\
\hline (3) Juno & 0.83 & 0.74 & 7.209531 & 244 & $\mathrm{~S}$ & 0.037 & 0.196 & 0.016 & -0.0064 \\
\hline (6) Hebe & 0.95 & 0.94 & 7.274470 & 192 & $\mathrm{~S}$ & 0.035 & 0.055 & 0.056 & -0.0048 \\
\hline (7) Iris & 0.89 & 0.80 & 7.138841 & 203 & S & 0.040 & 0.100 & 0.019 & -0.0053 \\
\hline (8) Flora & 0.94 & 0.89 & 12.86667 & 141 & S & .037 & 0.038 & 0.030 & -0.0037 \\
\hline (9) Metis & 0.84 & 0.75 & 5.079176 & 180 & S & 0.037 & 0.074 & 0.015 & -0.0034 \\
\hline (10) Hygiea & 0.78 & 0.98 & 27.623152 & 429 & $\mathrm{C}$ & 0.034 & 0.049 & 0.023 & -0.0037 \\
\hline (12) Victoria & 0.82 & 0.86 & 8.659852 & 117 & S & 047 & 0.062 & 0.012 & -0.0030 \\
\hline (15) Eunomia & 0.72 & 0.83 & 6.082752 & 272 & S & 0.047 & 0.058 & 0.049 & -0.0053 \\
\hline (16) Psyche & 0.85 & 0.96 & 4.195947 & 264 & $\mathrm{X}$ & 025 & 0.039 & 0.099 & -0.0046 \\
\hline (17) Thetis & 0.81 & 0.97 & 12.266025 & 93 & S & 0.038 & 0.041 & 0.023 & -0.0033 \\
\hline (18) Melpomene & 0.85 & 0.90 & 11.571433 & 148 & S & .038 & 0.055 & 0.021 & -0.0037 \\
\hline (19) Fortuna & 0.86 & 0.95 & 7.443223 & 220 & $\mathrm{C}$ & 0.026 & 0.034 & 0.112 & -0.0045 \\
\hline (20) Massalia & 0.86 & 0.74 & 8.09902 & 151 & S & 0.030 & & & \\
\hline (21) Lutetia & 0.87 & 0.80 & 8.16827 & 99 & $\mathrm{X}$ & 0.033 & 0.122 & 0.003 & -0.0023 \\
\hline (22) Kalliope & 0.78 & 0.95 & 4.148200 & 187 & $\mathrm{X}$ & 0.027 & 0.034 & 0.018 & -0.0025 \\
\hline (23) Thalia & 0.95 & 0.79 & 12.31240 & 111 & S & 0.028 & 0.035 & 0.031 & -0.0037 \\
\hline (29) Amphitrite & 0.93 & 1.00 & 5.390119 & 219 & S & 0.025 & 0.035 & 0.112 & -0.0045 \\
\hline (32) Pomona & 0.78 & 0.91 & 9.447659 & 82 & S & 0.028 & 0.044 & 0.023 & -0.0042 \\
\hline (39) Laetitia & 0.78 & 0.86 & 5.138237 & 159 & S & 0.029 & 0.051 & 0.056 & -0.0049 \\
\hline (41) Daphne & 0.83 & 0.82 & 5.98798 & 182 & $\mathrm{C}$ & 0.061 & & & \\
\hline (42) Isis & 0.93 & 0.93 & 13.597010 & 107 & S & 0.047 & 0.074 & 0.036 & -0.0061 \\
\hline (43) Ariadne & 0.66 & 0.76 & 5.761986 & 65 & S & 0.038 & 0.089 & 0.006 & -0.0034 \\
\hline (44) Nysa & 0.74 & 0.82 & 6.421417 & 73 & $\mathrm{X}$ & 0.038 & & & \\
\hline (45) Eugenia & 0.91 & 0.81 & 5.699152 & 214 & $\mathrm{C}$ & 0.021 & 0.062 & 0.013 & -0.0041 \\
\hline (52) Europa & 0.91 & 0.87 & 5.629958 & 312 & $\mathrm{C}$ & 0.029 & 0.052 & 0.066 & -0.0044 \\
\hline (54) Alexandra & 0.87 & 0.91 & 7.023337 & 171 & $\mathrm{C}$ & 0.035 & 0.030 & 0.145 & -0.0056 \\
\hline (55) Pandora & 0.93 & 0.97 & 4.804044 & 67 & $\mathrm{X}$ & 0.038 & 0.087 & 0.019 & -0.0054 \\
\hline (63) Ausonia & 0.60 & 0.97 & 9.297591 & 108 & $\mathrm{~S}$ & 0.023 & 0.056 & 0.010 & -0.0032 \\
\hline (69) Hesperia & 0.89 & 0.79 & 5.655199 & 143 & $\mathrm{X}$ & 0.039 & 0.080 & 0.011 & -0.0033 \\
\hline (79) Eurynome & 0.93 & 0.97 & 5.97857 & 68 & $\mathrm{~S}$ & 0.035 & 0.072 & 0.061 & -0.0056 \\
\hline (85) Io & 0.98 & 0.96 & 6.875110 & 157 & $\mathrm{C}$ & 0.033 & 0.028 & 0.175 & -0.0058 \\
\hline (87) Sylv & 0.76 & 0.91 & 5.183642 & 271 & $\mathrm{X}$ & 0.032 & 0.084 & 0.019 & -0.0055 \\
\hline (88) Thisbe & 0.96 & 0.84 & 6.041296 & 200 & $\mathrm{C}$ & 0.049 & 0.039 & 0.028 & -0.0033 \\
\hline (93) Minerva & 0.99 & 0.97 & 5.980877 & 146 & $\mathrm{C}$ & 0.027 & 0.024 & 0.211 & -0.0047 \\
\hline (107) Camilla & 0.75 & 0.98 & 4.84393 & 237 & $\mathrm{C}$ & 0.031 & 0.028 & 0.165 & -0.0051 \\
\hline
\end{tabular}




\begin{tabular}{|c|c|c|c|c|c|c|c|c|c|}
\hline Asteroid & $b / a$ & $c / b$ & $P(h)$ & $D(k m)$ & Tax & $r m s_{e}$ & $f_{1}$ & $f_{2}$ & $k$ \\
\hline (110) Lydia & 0.90 & 0.99 & 10.92580 & 89 & $\mathrm{X}$ & 0.058 & & & \\
\hline (115) Thyra & 0.93 & 0.87 & 7.23996 & 83 & $\mathrm{~S}$ & 0.051 & & & \\
\hline (125) Liberatrix & 0.97 & 0.94 & 3.968198 & 47 & $\mathrm{X}$ & 0.035 & & & \\
\hline (129) Antigone & 0.87 & 0.81 & 4.957154 & 125 & $\mathrm{X}$ & 0.030 & 0.156 & 0.009 & -0.0055 \\
\hline (130) Elektra & 0.85 & 0.90 & 5.224664 & 189 & $\mathrm{C}$ & 0.038 & 0.034 & 0.129 & -0.0059 \\
\hline (135) Hertha & 0.88 & 0.86 & 8.40061 & 82 & $\mathrm{X}$ & 0.025 & 0.089 & 0.009 & -0.0048 \\
\hline (158) Koronis & 0.75 & 0.68 & 14.20569 & 39 & $\mathrm{~S}$ & 0.060 & & & \\
\hline (165) Loreley & 0.98 & 0.91 & 7.226670 & 160 & $\mathrm{C}$ & 0.057 & & & \\
\hline (167) Urda & 0.84 & 0.93 & 13.06137 & 42 & $\mathrm{~S}$ & 0.039 & & & \\
\hline (173) Ino & 0.96 & 0.91 & 6.116510 & 159 & $\mathrm{C}$ & 0.041 & & & \\
\hline (192) Nausikaa & 0.91 & 0.89 & 13.62170 & 107 & $\mathrm{~S}$ & 0.037 & 0.048 & 0.086 & -0.0052 \\
\hline (196) Philomela & 0.98 & 1.00 & 8.332826 & 146 & S & 0.010 & & & \\
\hline (201) Penelope & 0.87 & 0.90 & 3.747454 & 70 & $\mathrm{X}$ & 0.028 & 0.073 & 0.029 & -0.0051 \\
\hline (208) Lacrimosa & 0.85 & 0.76 & 14.0769 & 44 & $\mathrm{~S}$ & 0.040 & & & \\
\hline (218) Bianca & 0.60 & 0.65 & 6.337172 & 62 & S & 0.110 & & & \\
\hline (230) Athamantis & 0.90 & 0.98 & 23.984501 & 113 & $\mathrm{~S}$ & 0.040 & 0.061 & 0.069 & -0.0060 \\
\hline (250) Bettina & 0.83 & 0.79 & 5.05442 & 85 & $\mathrm{X}$ & 0.039 & 0.062 & 0.052 & -0.0054 \\
\hline (277) Elvira & 0.69 & 0.91 & 29.6922 & 29 & $\mathrm{~S}$ & 0.059 & & & \\
\hline (283) Emma & 0.67 & 0.97 & 6.89522 & 150 & $\mathrm{X}$ & 0.015 & & & \\
\hline (306) Unitas & 0.81 & 0.88 & 8.73875 & 49 & $\mathrm{~S}$ & 0.035 & & & \\
\hline (311) Claudia & 0.55 & 0.87 & 7.53139 & 27 & S & 0.038 & & & \\
\hline (321) Florentina & 0.72 & 0.77 & 2.870866 & 31 & $\mathrm{~S}$ & 0.025 & & & \\
\hline (337) Devosa & 0.92 & 0.66 & 4.653680 & 63 & $\mathrm{X}$ & 0.033 & 0.139 & 0.019 & -0.0049 \\
\hline (349) Dembowska & 0.82 & 0.75 & 4.701211 & 143 & $\mathrm{~S}$ & 0.040 & 0.094 & 0.005 & -0.0029 \\
\hline (354) Eleonora & 0.91 & 0.98 & 4.277185 & 162 & $\mathrm{~S}$ & 0.044 & 0.046 & 0.020 & -0.0030 \\
\hline (372) Palma & 0.96 & 0.92 & 8.591030 & 195 & $\mathrm{C}$ & 0.040 & 0.059 & 0.033 & -0.0048 \\
\hline (376) Geometria & 0.94 & 0.97 & 7.715890 & 37 & S & 0.041 & 0.092 & 0.043 & -0.0048 \\
\hline (382) Dodona & 0.72 & 0.78 & 4.113226 & 60 & $\mathrm{X}$ & 0.056 & & & \\
\hline (423) Diotima & 0.93 & 0.99 & 4.775377 & 217 & $\mathrm{C}$ & 0.029 & & & \\
\hline (451) Patientia & 0.98 & 0.97 & 9.74119 & 230 & $\mathrm{C}$ & 0.045 & & & \\
\hline (511) Davida & 0.90 & 0.90 & 5.129367 & 337 & $\mathrm{C}$ & 0.026 & 0.034 & 0.122 & -0.0046 \\
\hline (532) Herculina & 0.87 & 0.99 & 9.404951 & 231 & S & 0.028 & 0.057 & 0.052 & -0.0049 \\
\hline (534) Nassovia & 0.81 & 0.72 & 9.46895 & 37 & $\mathrm{~S}$ & 0.052 & & & \\
\hline (584) Semiramis & 0.93 & 0.70 & 5.068919 & 56 & S & 0.045 & 0.071 & 0.017 & -0.0037 \\
\hline (624) Hektor & 0.46 & 0.84 & 6.920509 & & $\mathrm{D}$ & 0.035 & & & \\
\hline (665) Sabine & 0.75 & 0.82 & 4.294032 & 56 & & 0.042 & & & \\
\hline (675) Ludmilla & 0.96 & 0.81 & 7.715486 & 350 & S & 0.029 & & & \\
\hline (690) Wratislavia & 0.89 & 0.70 & 8.61798 & 140 & $\mathrm{C}$ & 0.036 & & & \\
\hline (694) Ekard & 0.89 & 0.88 & 5.92193 & 92 & $\mathrm{C}$ & 0.041 & 0.086 & 0.033 & -0.0062 \\
\hline (720) Bohlinia & 0.76 & 0.73 & 8.91862 & 37 & $\mathrm{~S}$ & 0.054 & & & \\
\hline (776) Berbericia & 0.89 & 0.99 & 7.66701 & & $\mathrm{C}$ & 0.031 & 0.101 & 0.013 & -0.0051 \\
\hline (944) Hidalgo & 0.43 & 0.72 & 10.05863 & & $\mathrm{D}$ & 0.179 & & & \\
\hline (1036) Ganymed & 0.99 & 0.84 & 10.312 & 41 & $\mathrm{~S}$ & 0.036 & 0.047 & 0.062 & -0.0048 \\
\hline
\end{tabular}




\section{ACCEPTED MANUSCRIP'}

\begin{tabular}{llllcccccc}
\hline Asteroid & $b / a$ & $c / b$ & $P(h)$ & $D(k m)$ & Tax & $r m s_{e}$ & $f_{1}$ & $f_{2}$ & $k$ \\
\hline (1580) Betulia & 0.88 & 0.72 & 6.13836 & & $\mathrm{C}$ & 0.057 & & & \\
(1627) Ivar & 0.53 & 0.85 & 4.795170 & & $\mathrm{~S}$ & 0.031 & & & \\
(1862) Apollo & 0.83 & 0.66 & 3.065142 & & $\mathrm{~S}$ & 0.033 & & & \\
(1980) Tezcatlipoca & 0.66 & 0.74 & 7.25226 & & & 0.041 & & & \\
(2100) Ra-shalom & 0.86 & 0.89 & 19.820101 & & $\mathrm{C}$ & 0.038 & & & \\
(3908) Nyx & 0.84 & 0.86 & 4.42601 & & & 0.049 & & & \\
(5587) 1990 SB & 0.50 & 0.89 & 5.05227 & & & 0.025 & & & \\
(6053) 1993 BW3 & 0.94 & 0.82 & 2.5739 & & & 0.023 & 0.041 & 0.096 & -0.0049
\end{tabular}




\section{Figure captions}

Figure 1: Finding the overall dimensions of the shape. a) extent $(2 c)$ in spin axis $(z)$ direction. b) largest extent $(2 a)$ in $x y$-plane and largest extent $(2 b)$ in $x y$-plane, perpendicular to $a$. The images are from equatorial and polar viewing directions, respectively.

Figure 2: Previously unpublished lightcurve data of a) (31) Euphrosyne, b) (54) Alexandra, c) (79) Eurynome, d) (93) Minerva, e) (130) Elektra, f) (376) Geometria, g) (471) Papagena, and h) (776) Berbericia. The horizontal axes refer to the time (UT/hours) of observation; the observing date is given on each plot.

Figure 3: Period uncertainty of the eight convex inversion solutions published in this paper, versus the number of observed lightcurves.

Figure 4: Distribution of possible spin states of (31) Euphrosyne. a) rms of the model fit versus the rotation period $(P)$ and b) heliocentric ecliptic pole longitude $(\lambda)$ versus pole latitude $(\beta)$.

Figure 5: Shape model of (79) Eurynome from two perpendicular equatorial viewing directions.

Figure 6: Shape model of (93) Minerva from two perpendicular equatorial viewing directions.

Figure 7: Shape model of (130) Elektra from two perpendicular equatorial viewing directions.

Figure 8: Shape model of (376) Geometria from two perpendicular equatorial viewing directions.

Figure 9: Shape model of (776) Berbericia from two perpendicular equatorial viewing directions.

Figure 10: Comparison of different methods for characterizing the shapes of the objects. a) OD and E1, b) E1 and E2, and c) E2 and PMI.

Figure 11: Distributions of axis ratios of 87 convex inversion shape solutions from the a) E2 and b) PMI techniques. Different taxonomic types are plotted with different symbols as $\triangle=\mathrm{C}, \square=\mathrm{D},+=\mathrm{S}, \diamond=\mathrm{X}$, and $\times=$ undefined.

Figure 12: Comparing $b / a$ to the rotation period of the asteroid. Dimensions are from the E2 technique. Different taxonomic types are plotted with different symbols as $\triangle=\mathrm{C}$, $\square=\mathrm{D},+=\mathrm{S}, \diamond=\mathrm{X}$, and $\times=$ undefined.

Figure 13: Comparing $b / a$ to the size of the asteroid. Dimensions are from the E2 technique. Different taxonomic types are plotted with different symbols as $\triangle=\mathrm{C}, \square=\mathrm{D},+=\mathrm{S}, \diamond=\mathrm{X}$, and $\times=$ undefined. 
Figure 14: Angle $\theta$ between the rotation axis of the model and the axis of the largest moment of inertia. $\theta$ is plotted versus $b / a$ and $c / b$ (from the E2 method). Different taxonomic types are plotted with different symbols as $\triangle=\mathrm{C}, \square=\mathrm{D},+=\mathrm{S}, \diamond=\mathrm{X}$, and $\times=$ undefined.

Figure 15: rms of the E2 fit as a function of a) $b / a$ and $c / b$, and b) size. Different taxonomic types are plotted with different symbols as $\triangle=\mathrm{C}, \square=\mathrm{D},+=\mathrm{S}, \diamond=\mathrm{X}$, and $\times=$ undefined.

Figure 16: Fraction of the facets with area more than $20 \%$ of the largest facet area $\left(f_{1}\right)$ compared to the dimensions of the asteroid. Different taxonomic types are plotted with different symbols as $\triangle=\mathrm{C},+=\mathrm{S}, \diamond=\mathrm{X}$, and $\times=$ undefined.

Figure 17: Distribution of facet areas on model of (2) Pallas, (6) Hebe, and (776) Berbericia. The logarithms of the fractions of the largest facets from the total surface area are plotted in decreasing order.

Figure 18: Slope $k$ of the $\log \left(A / A_{t o t}\right)$ vs. size distribution. Different taxonomic types are plotted with different symbols as $\triangle=\mathrm{C},+=\mathrm{S}$, and $\diamond=\mathrm{X}$.

Figure 19: Fraction of the largest facets $\left(f_{1}\right)$ versus the deviation of the rotation axis from the axis of the largest moment of inertia. 


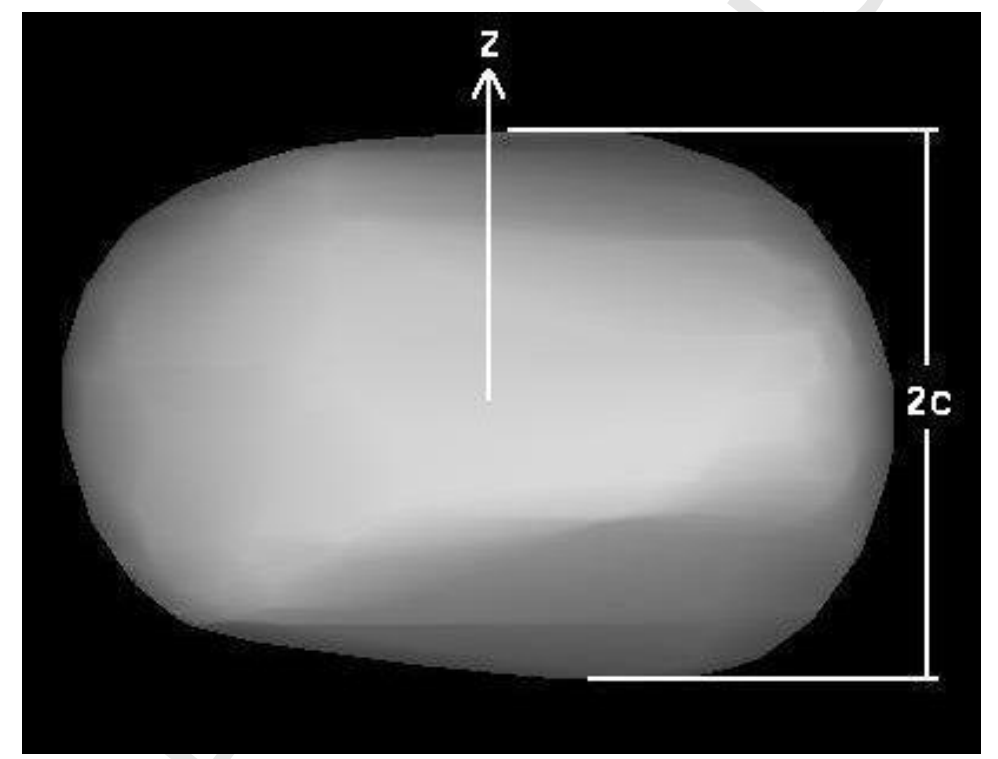

I10268_fig_1a 


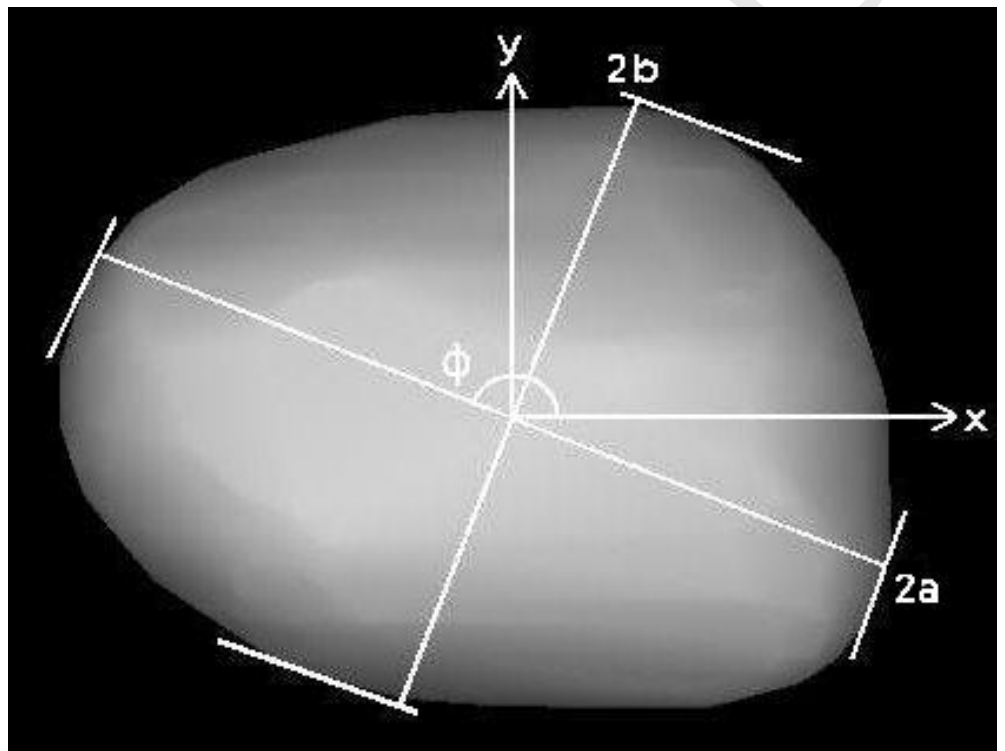




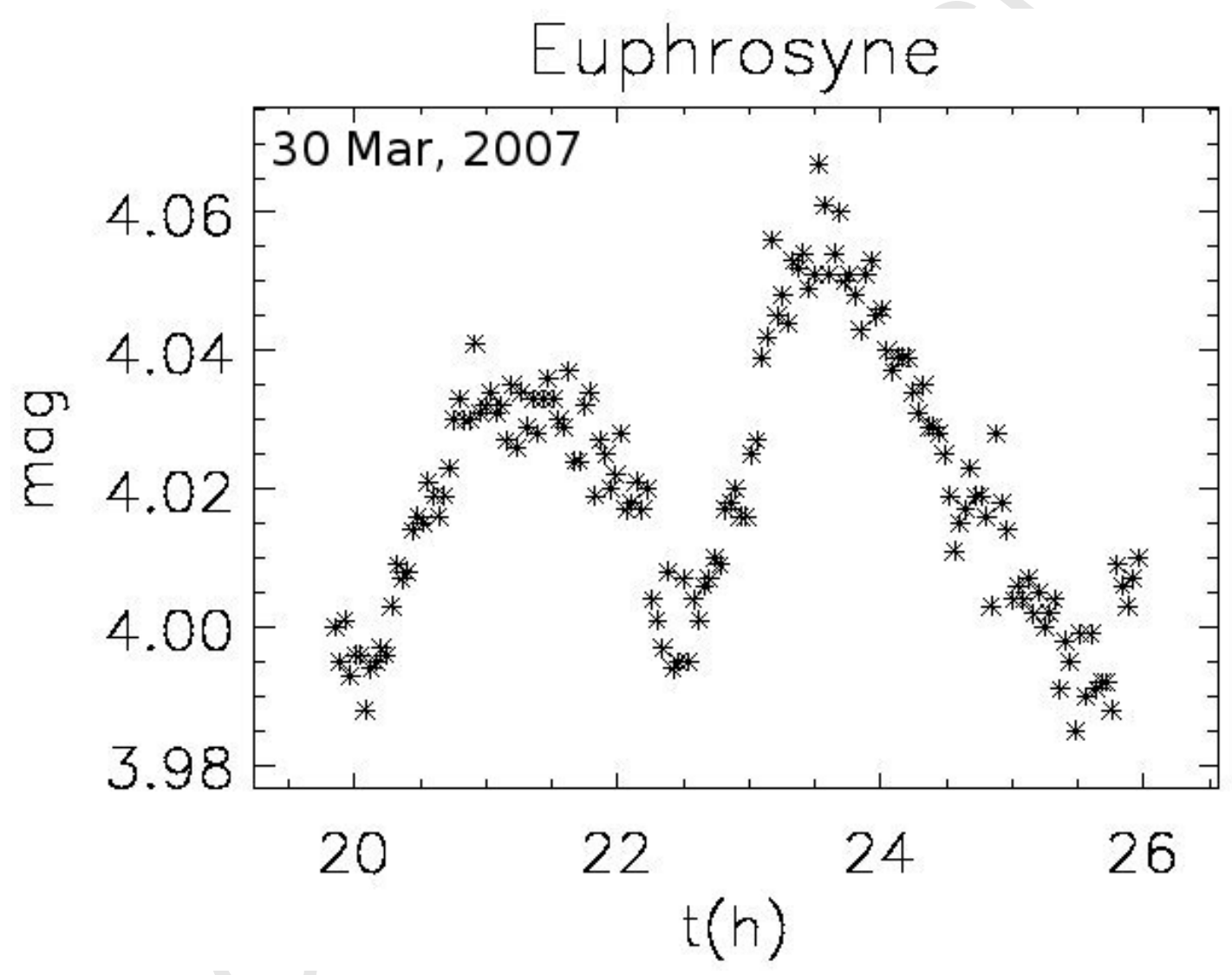



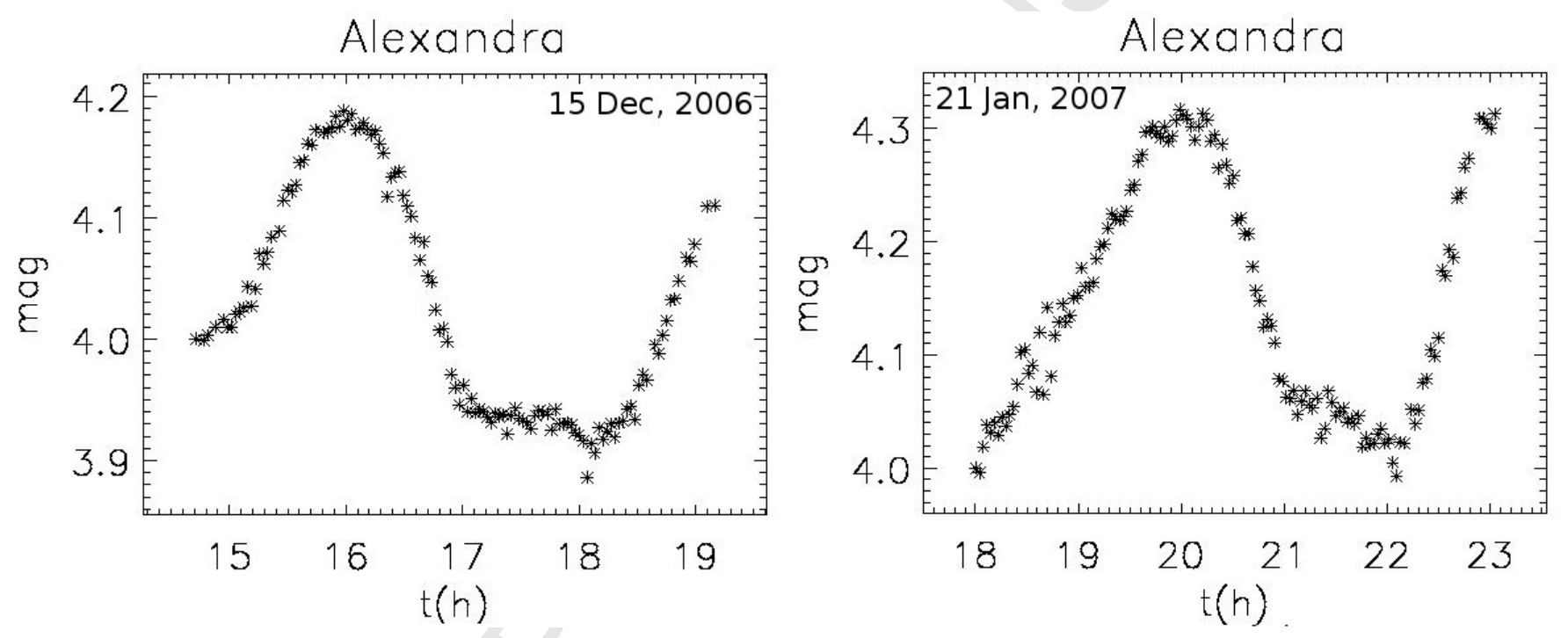


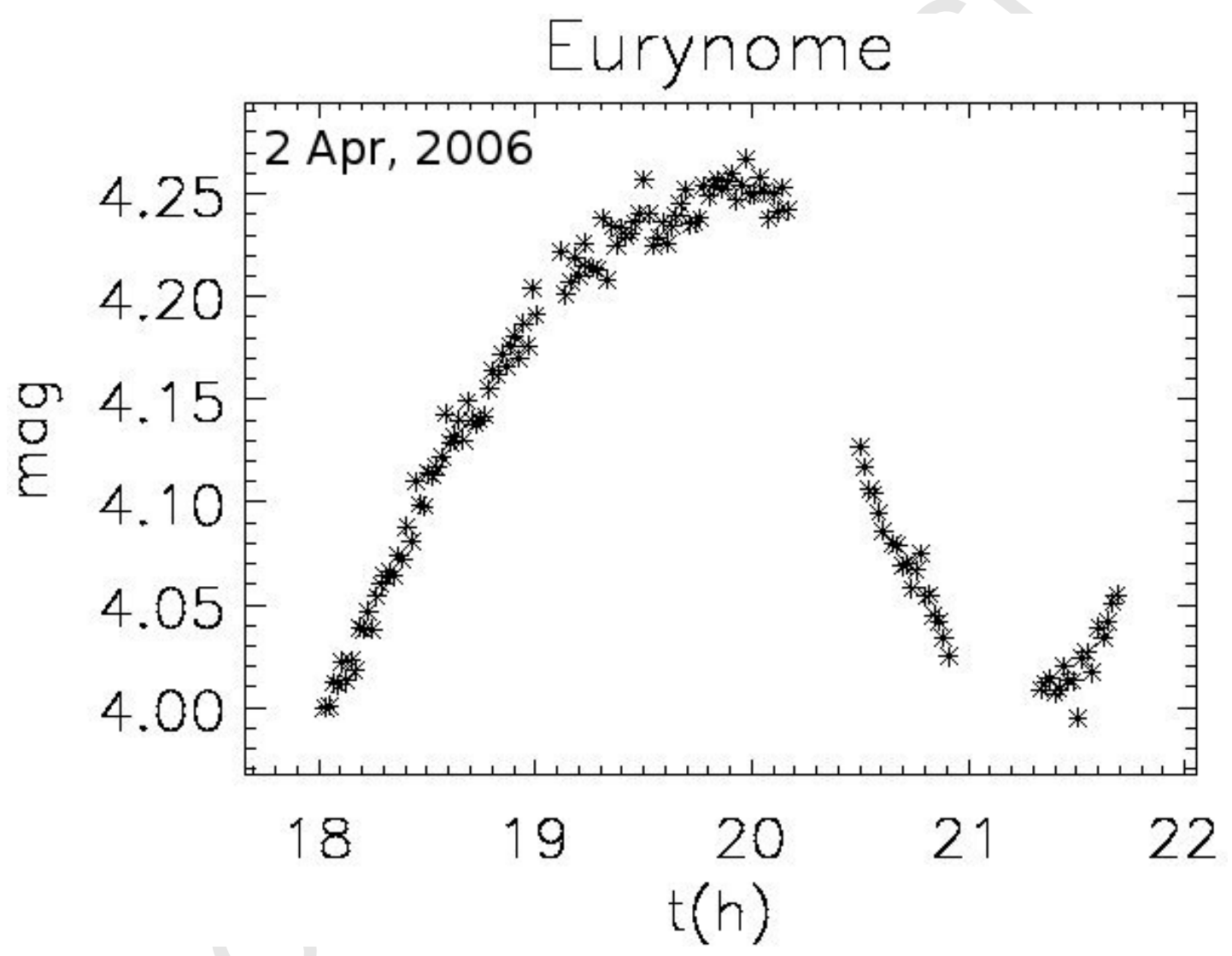



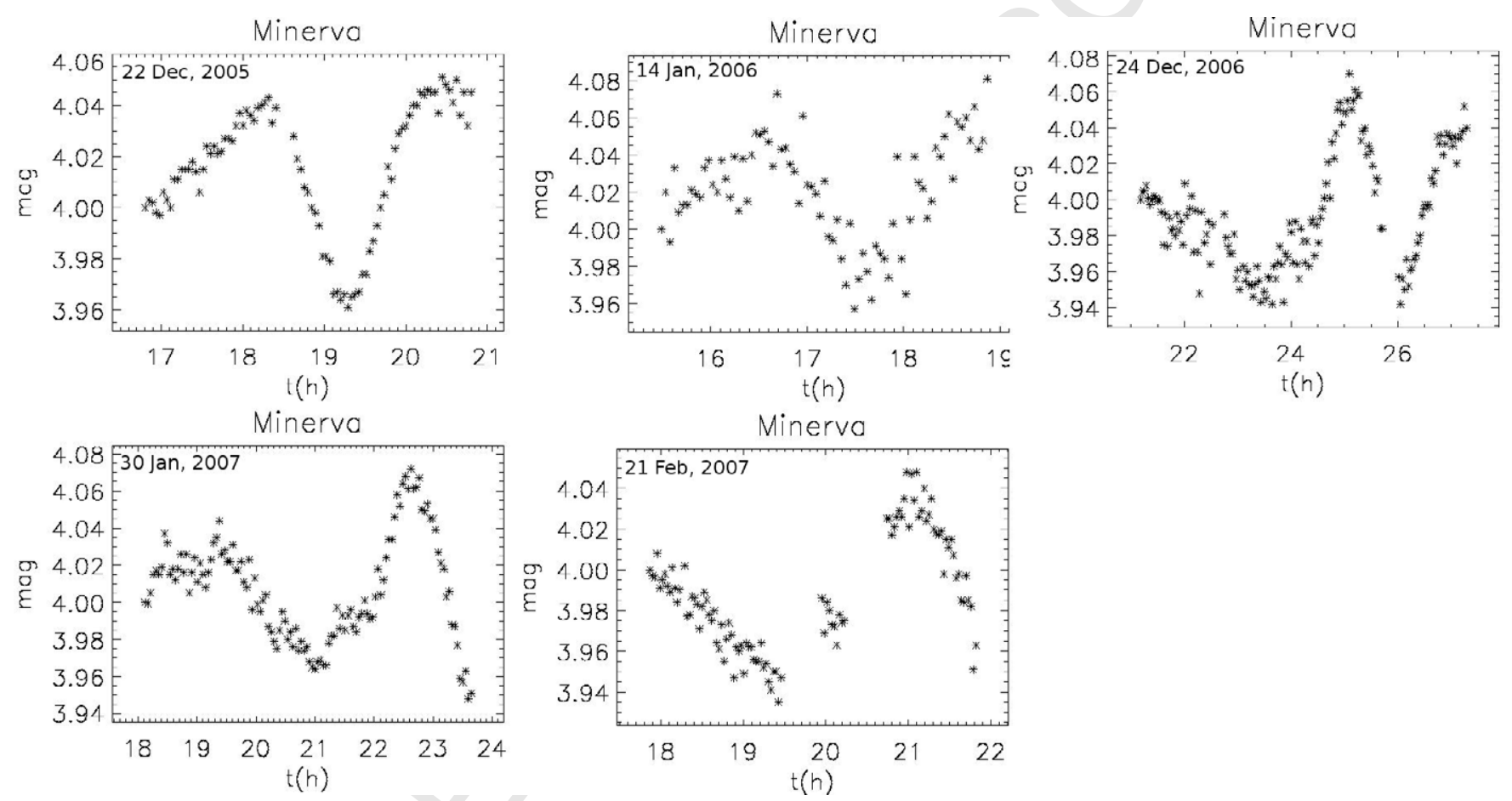

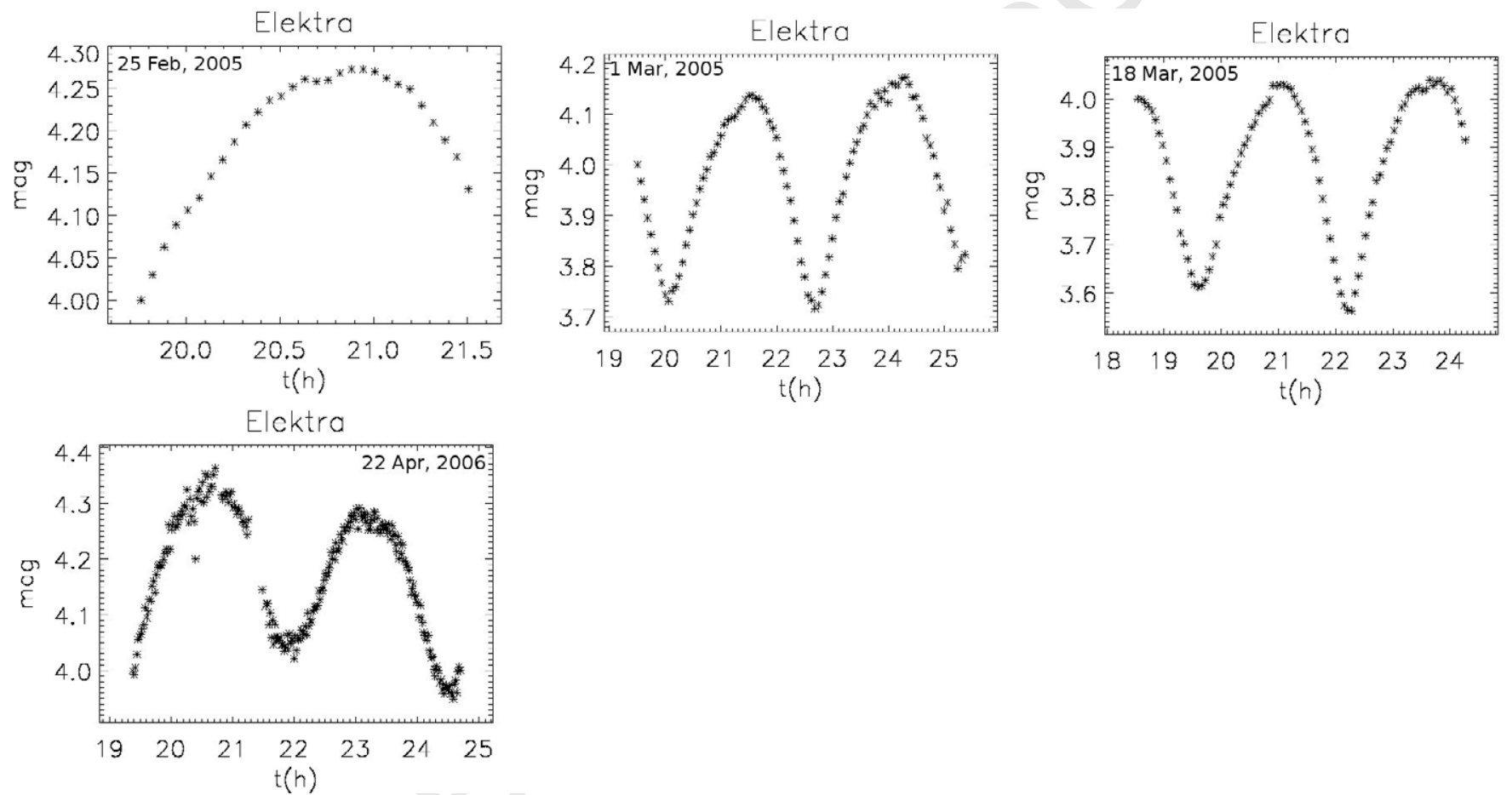


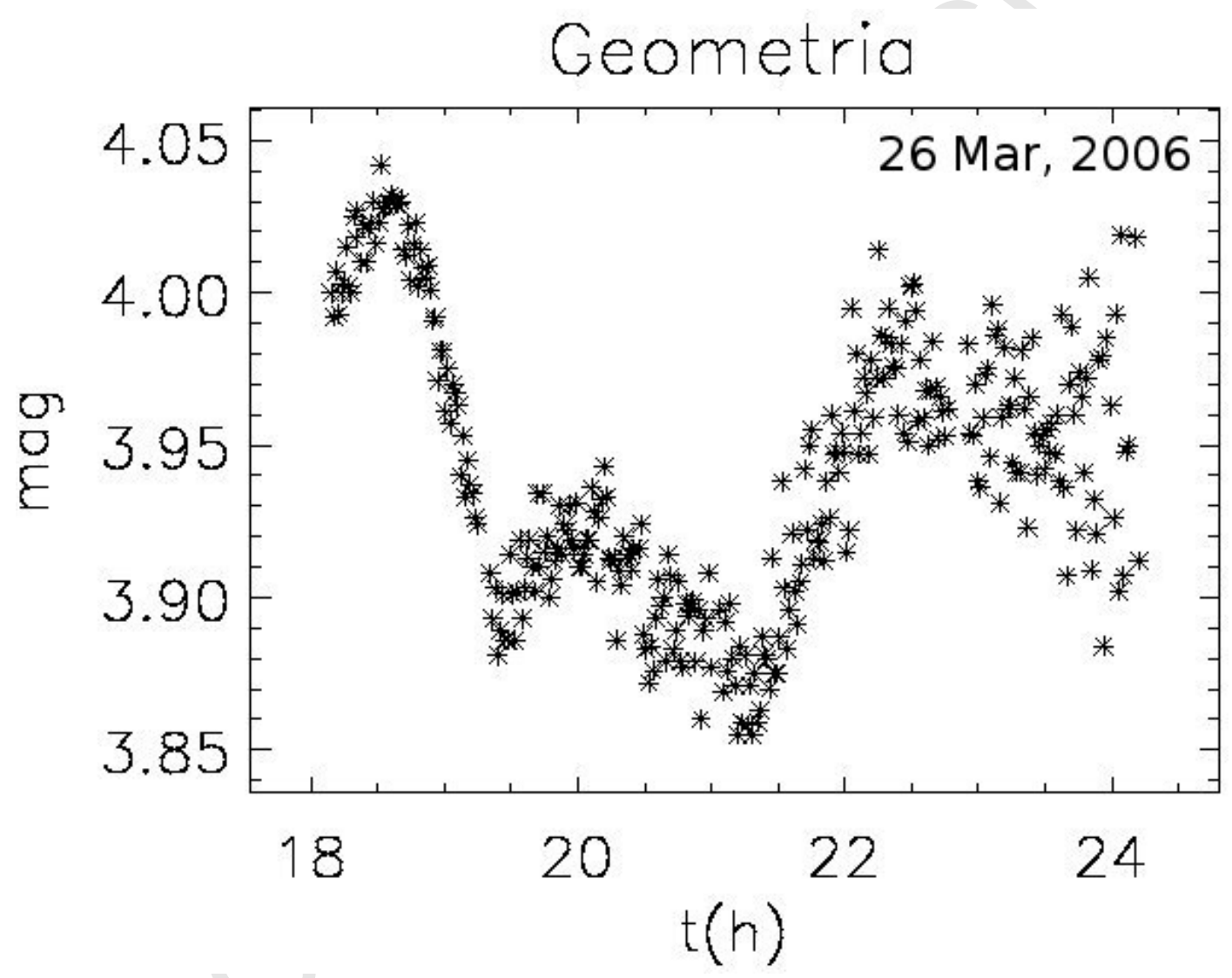




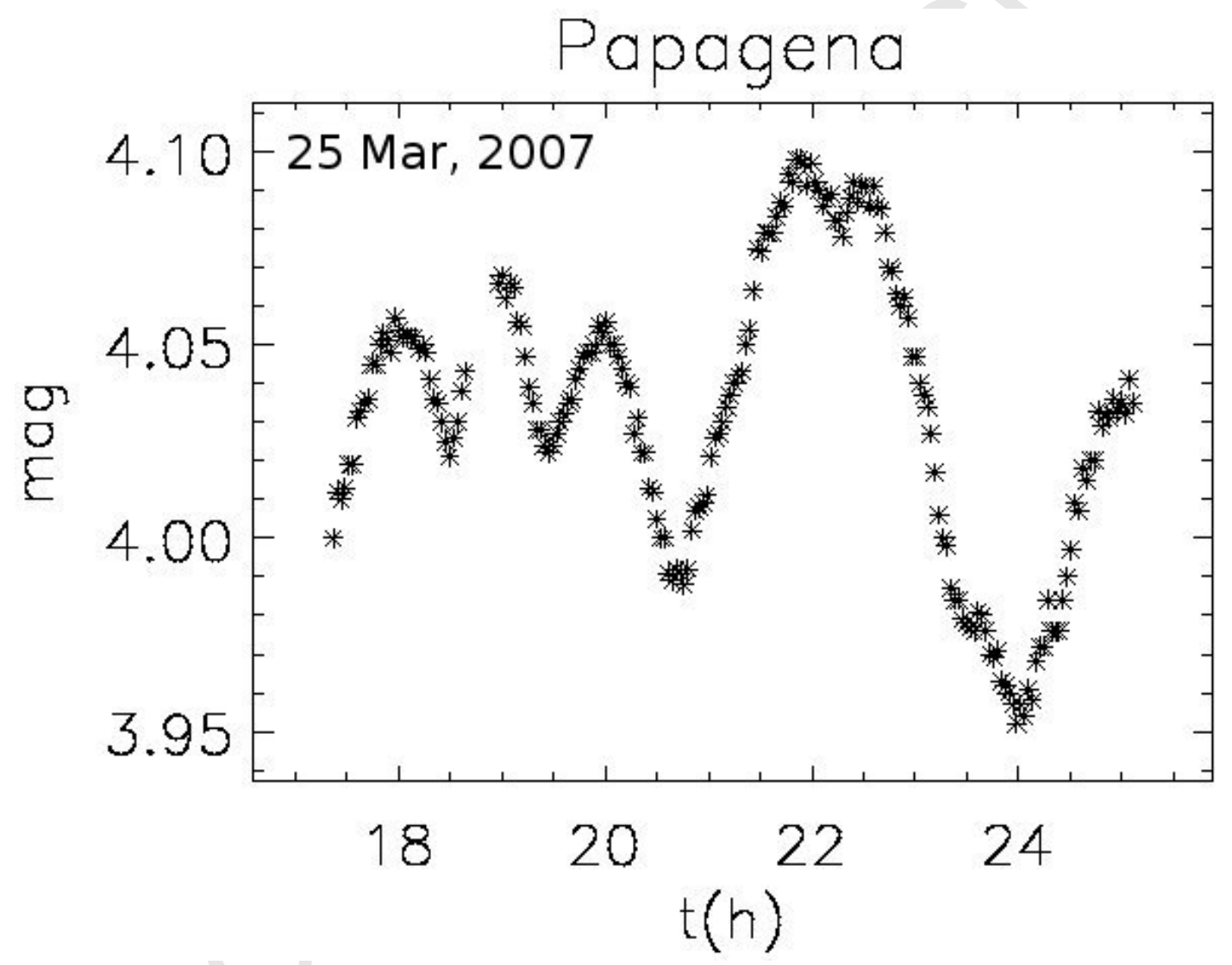



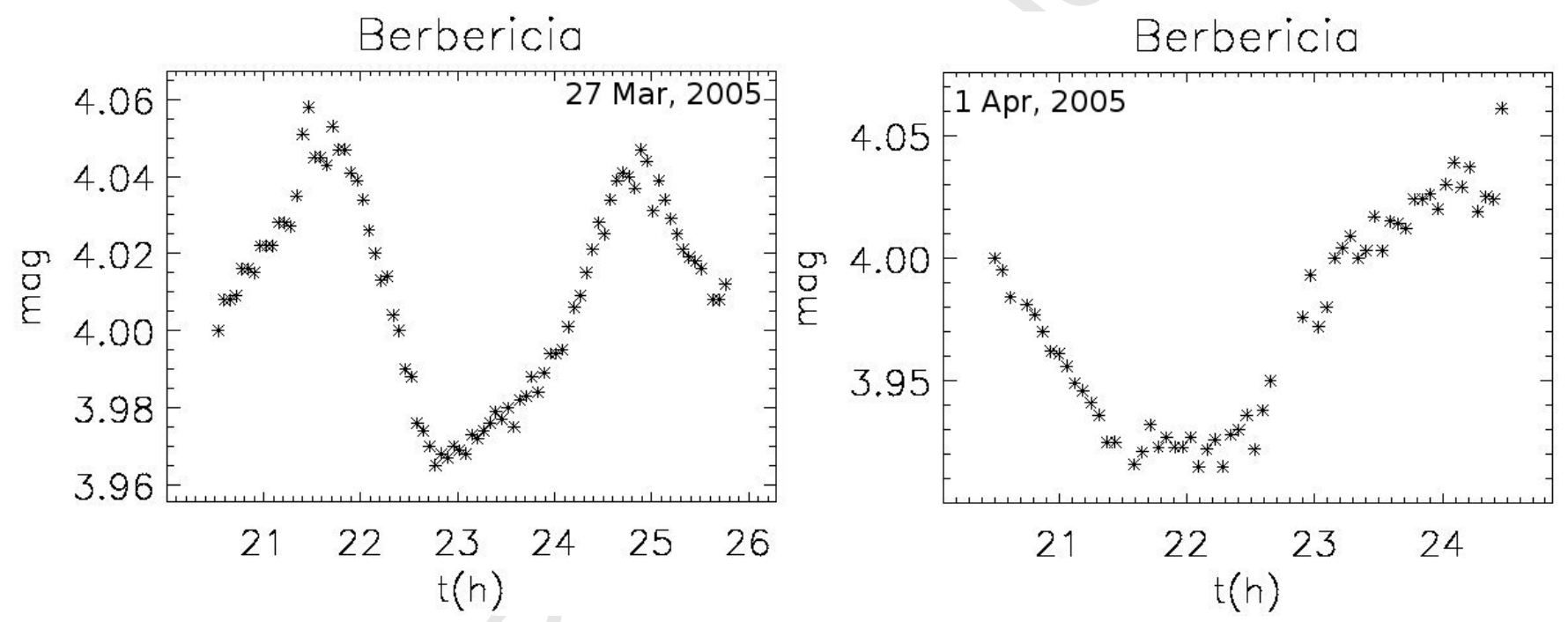


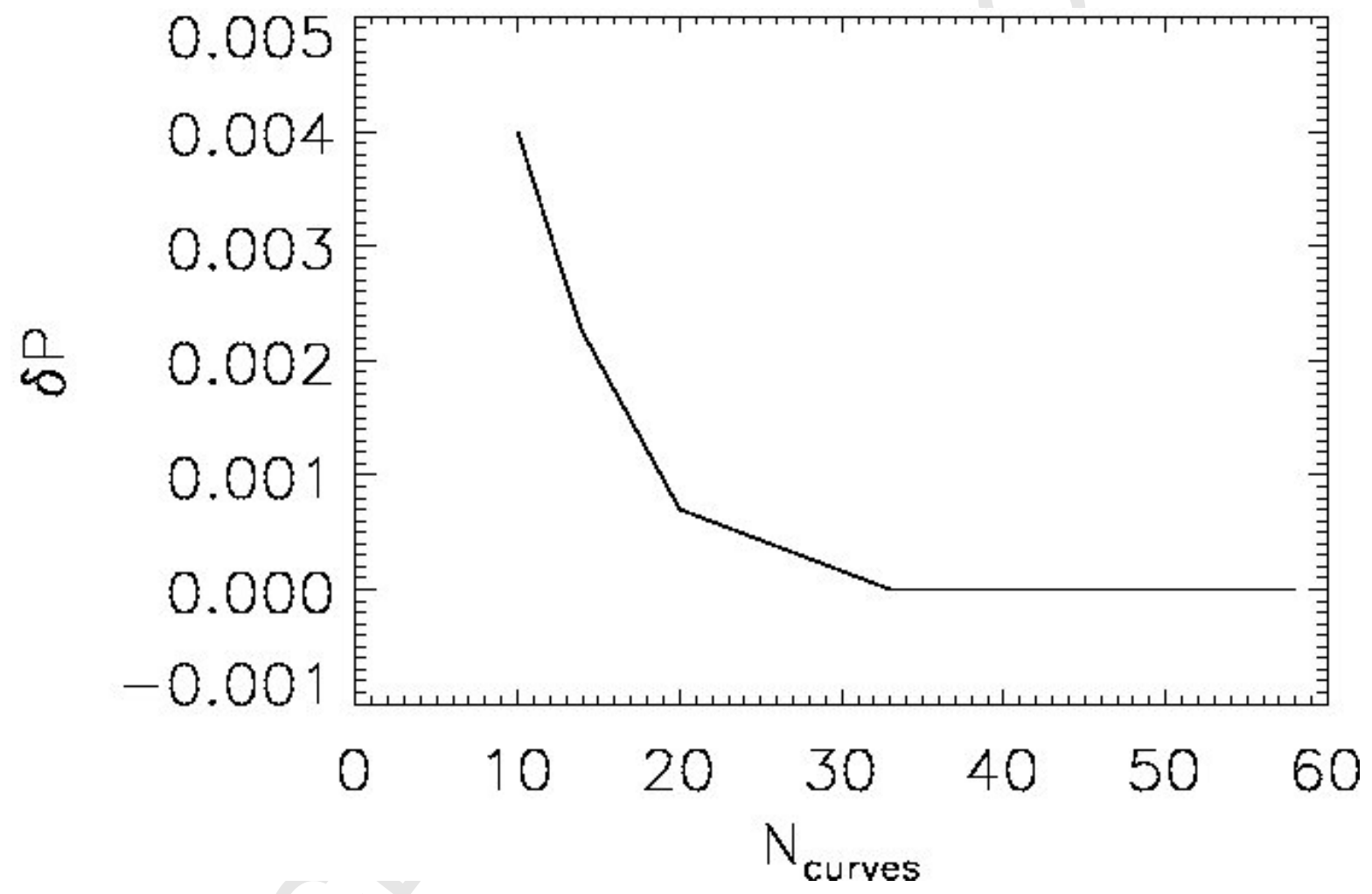




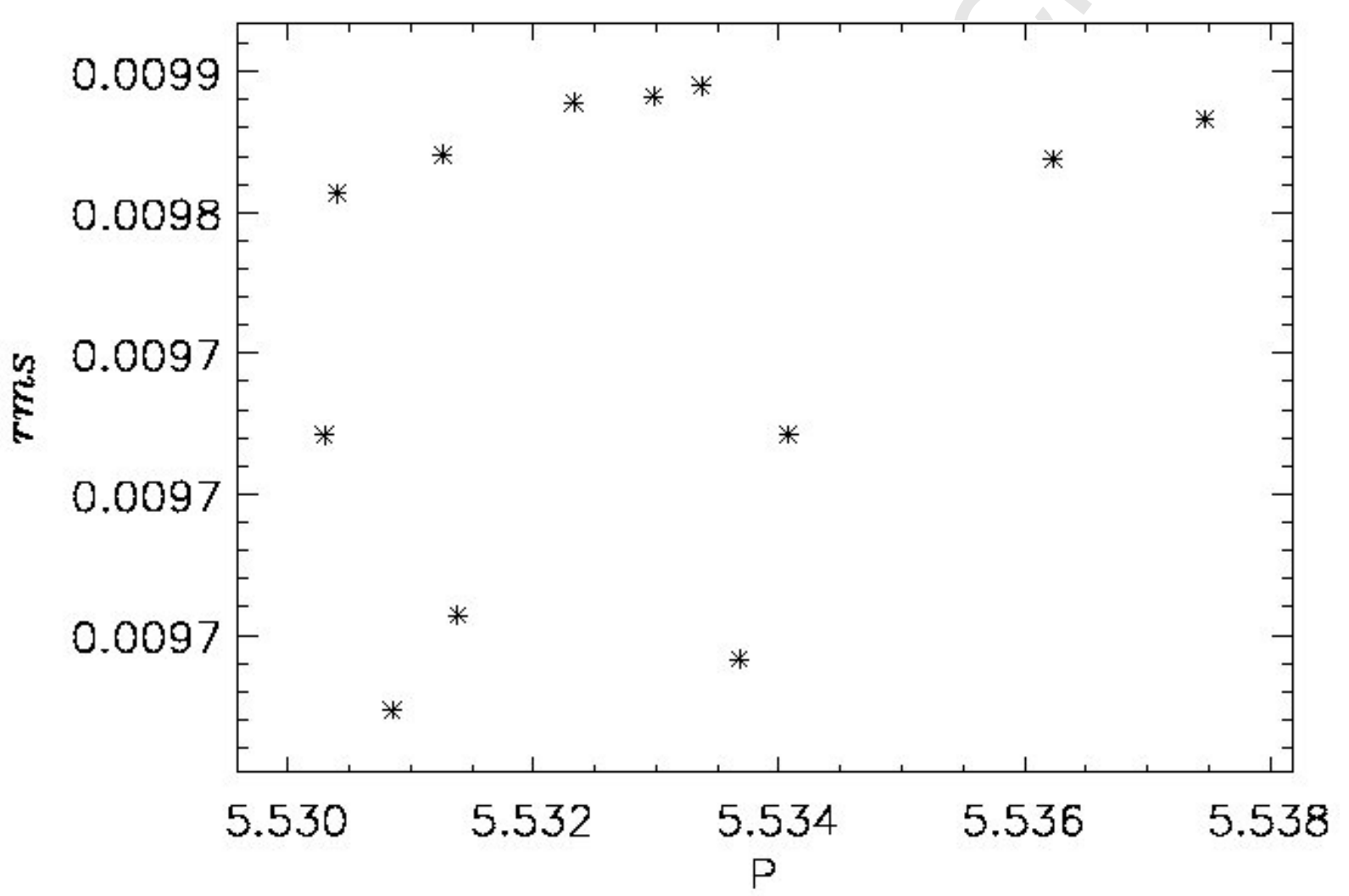




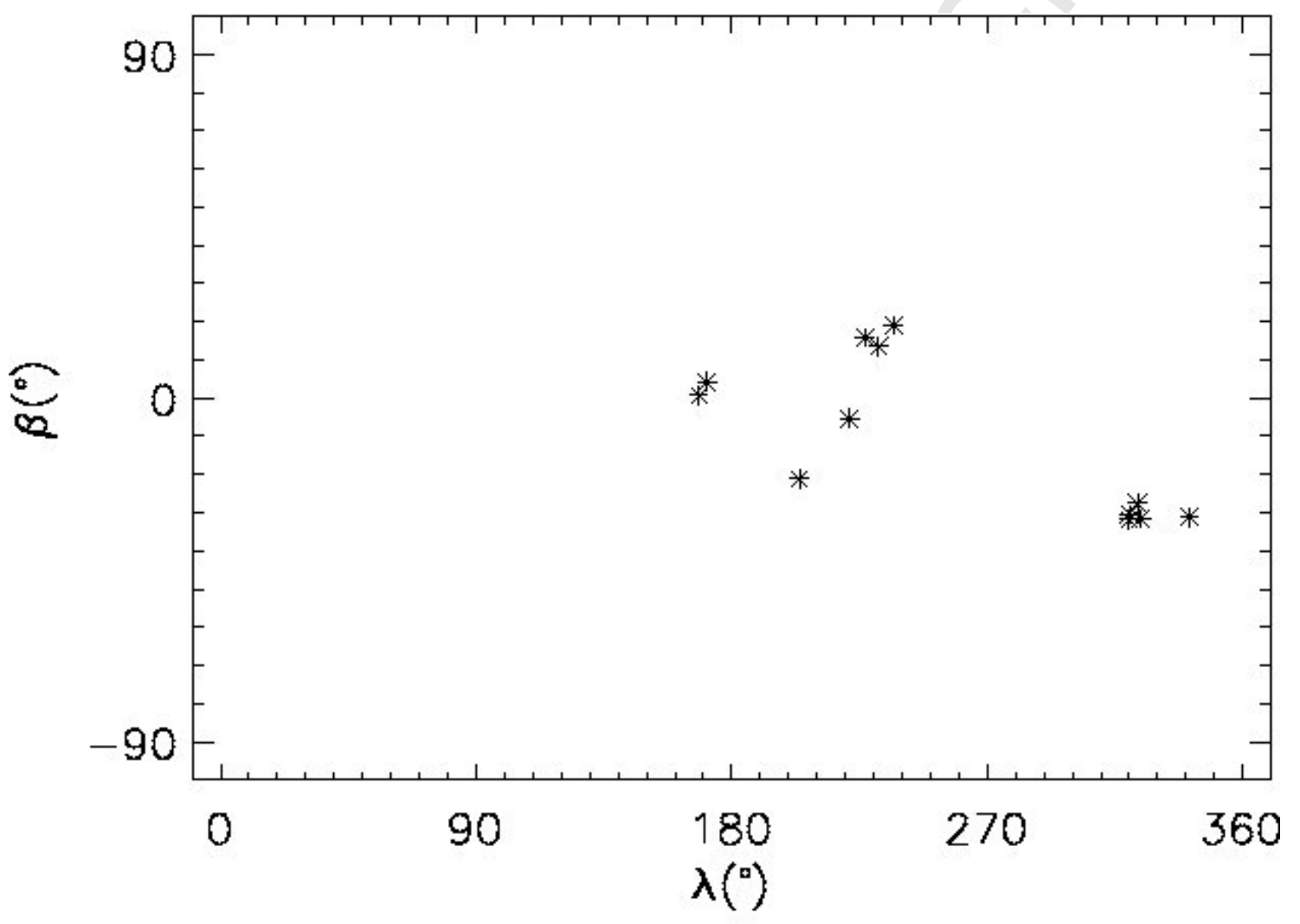




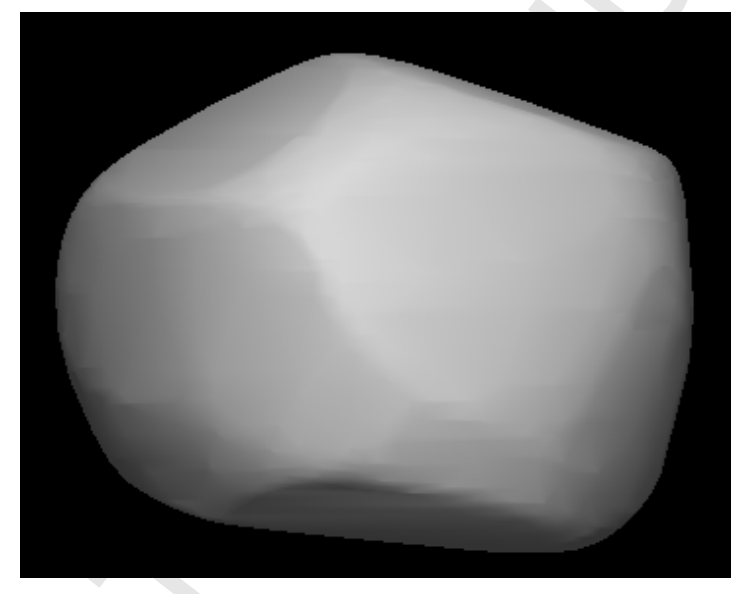




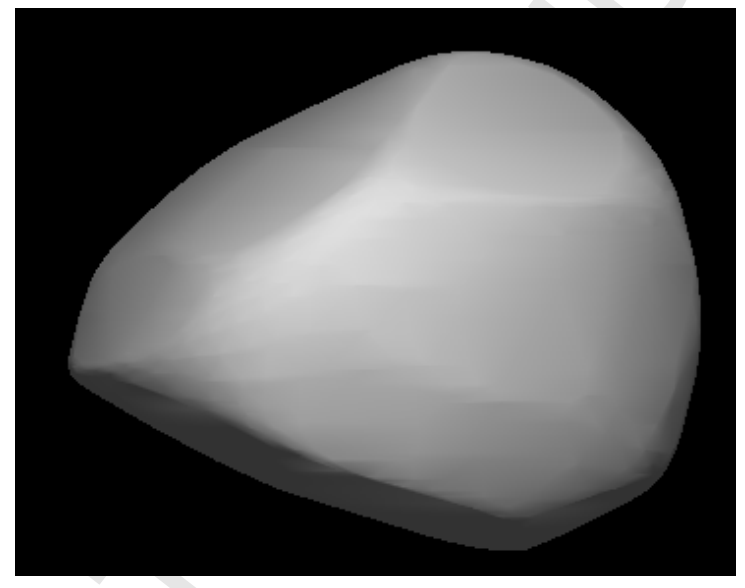

I10268_fig_5b 


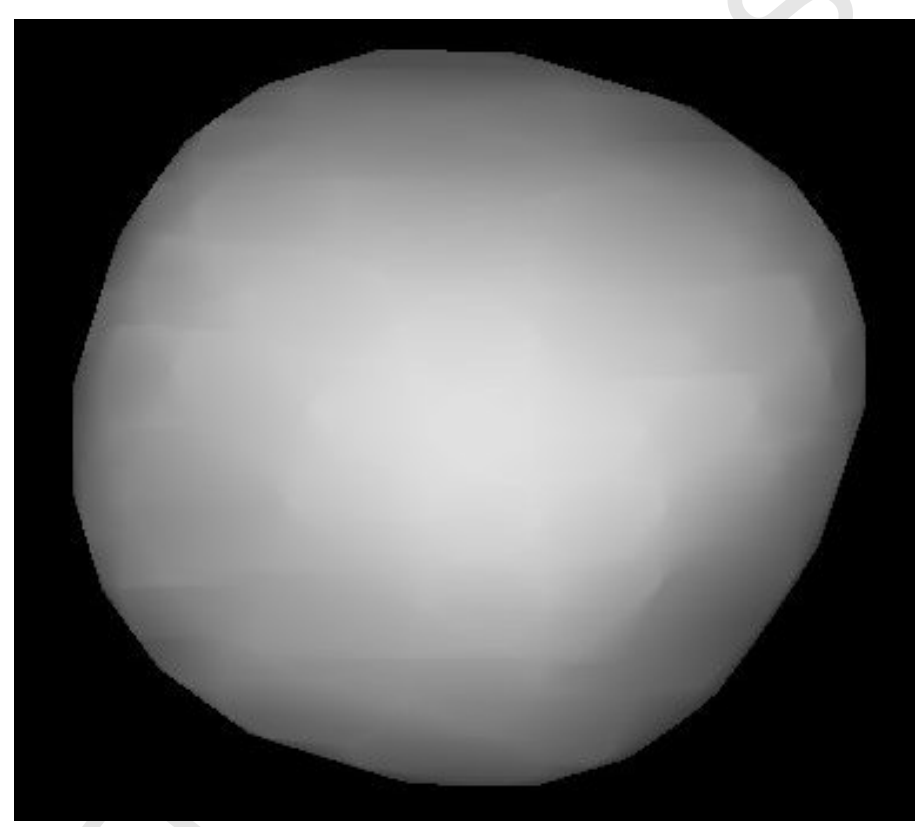




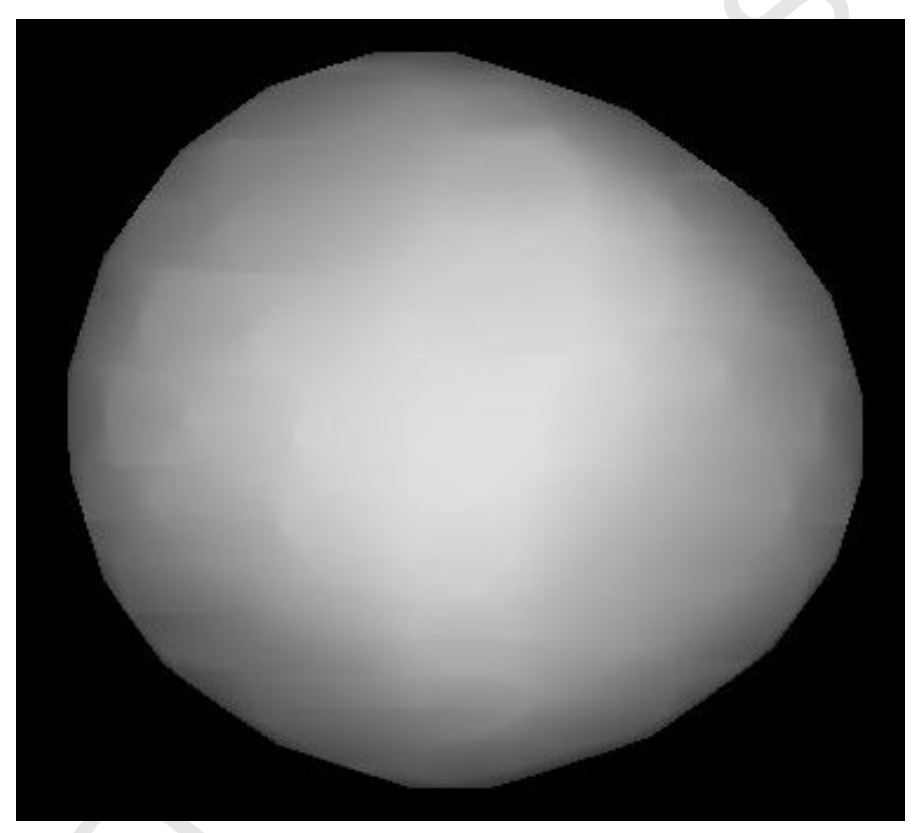




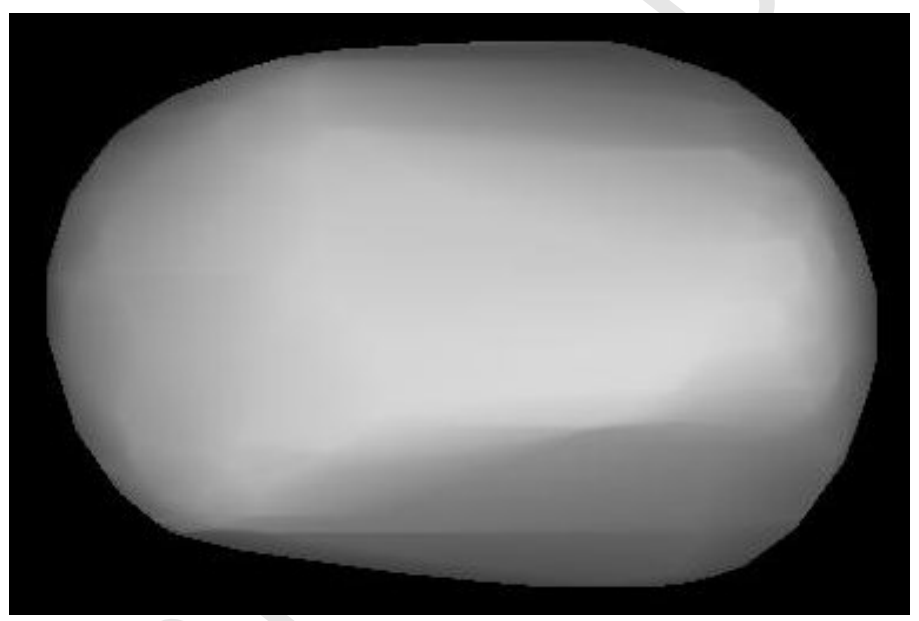

I10268_fig_7a 


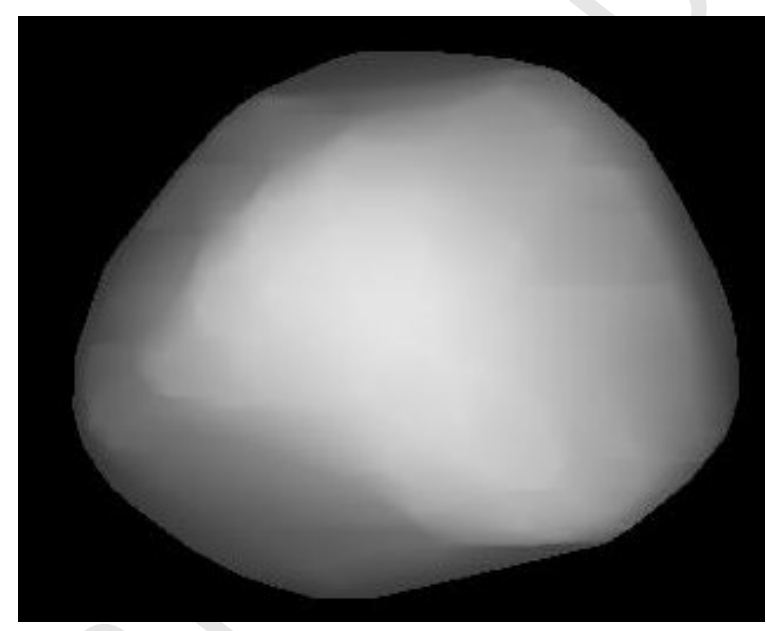

I10268_fig_7b 


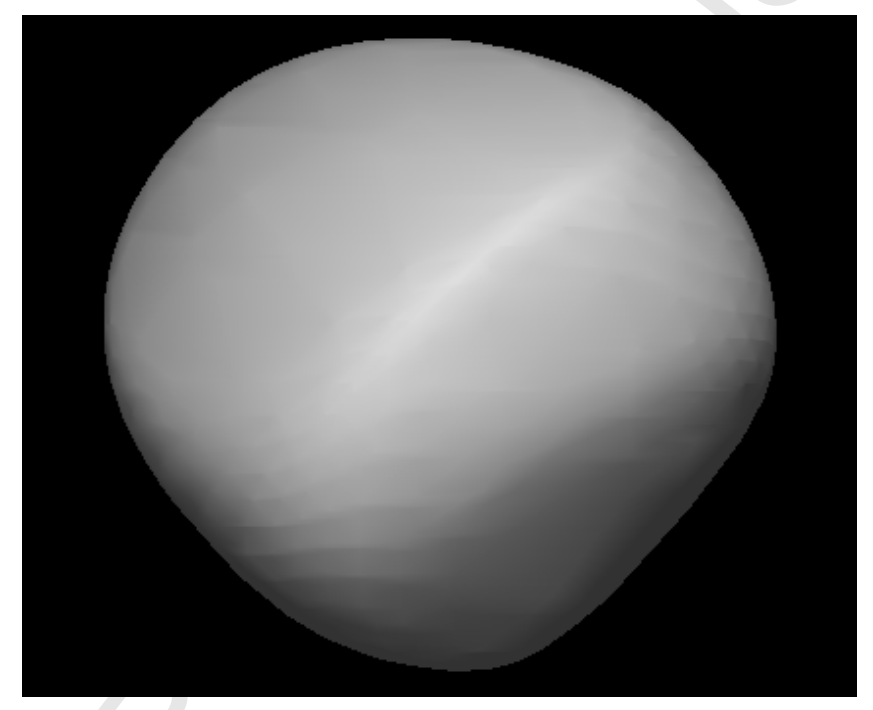




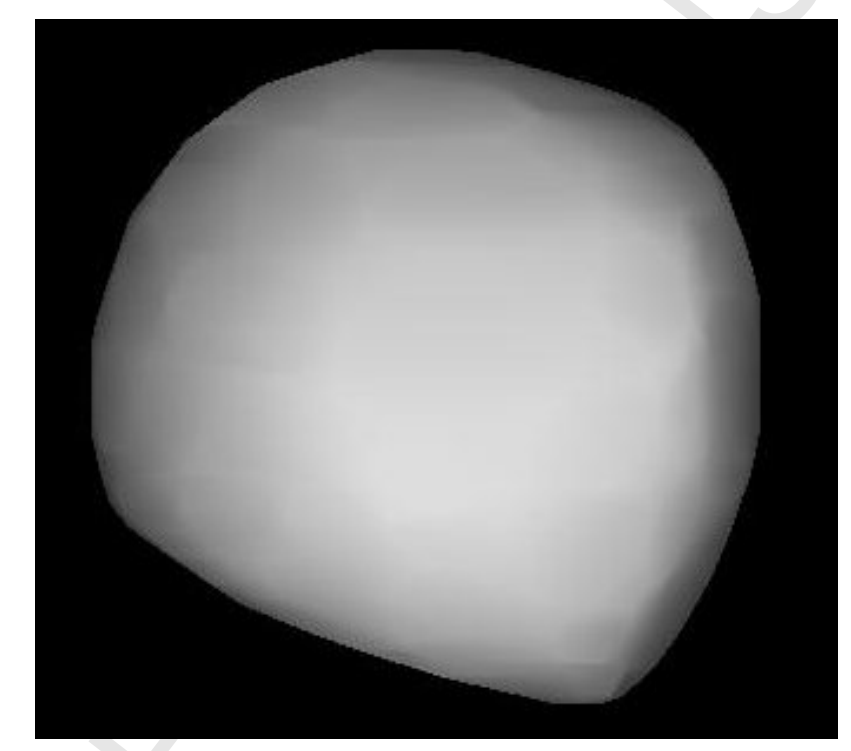



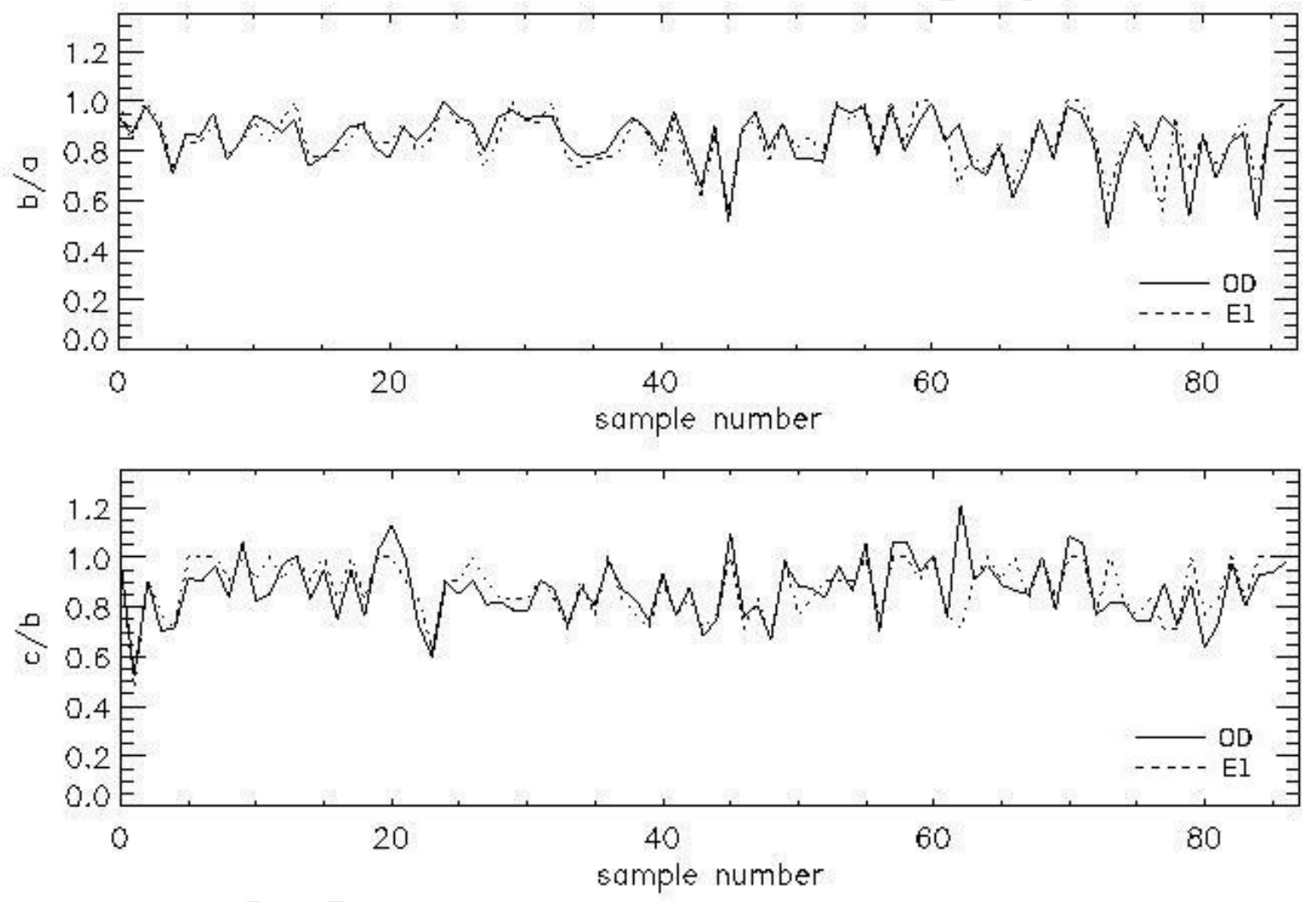

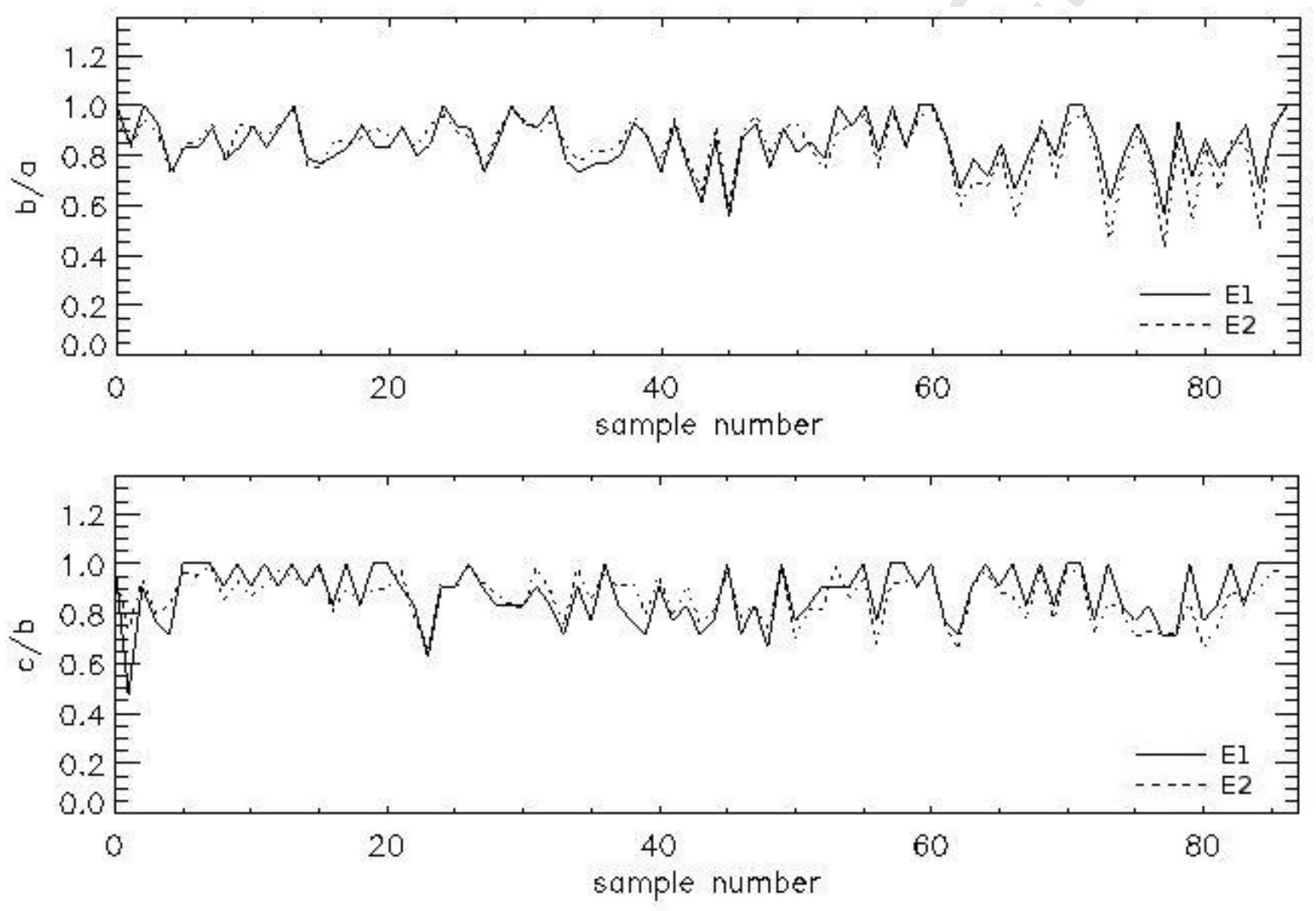

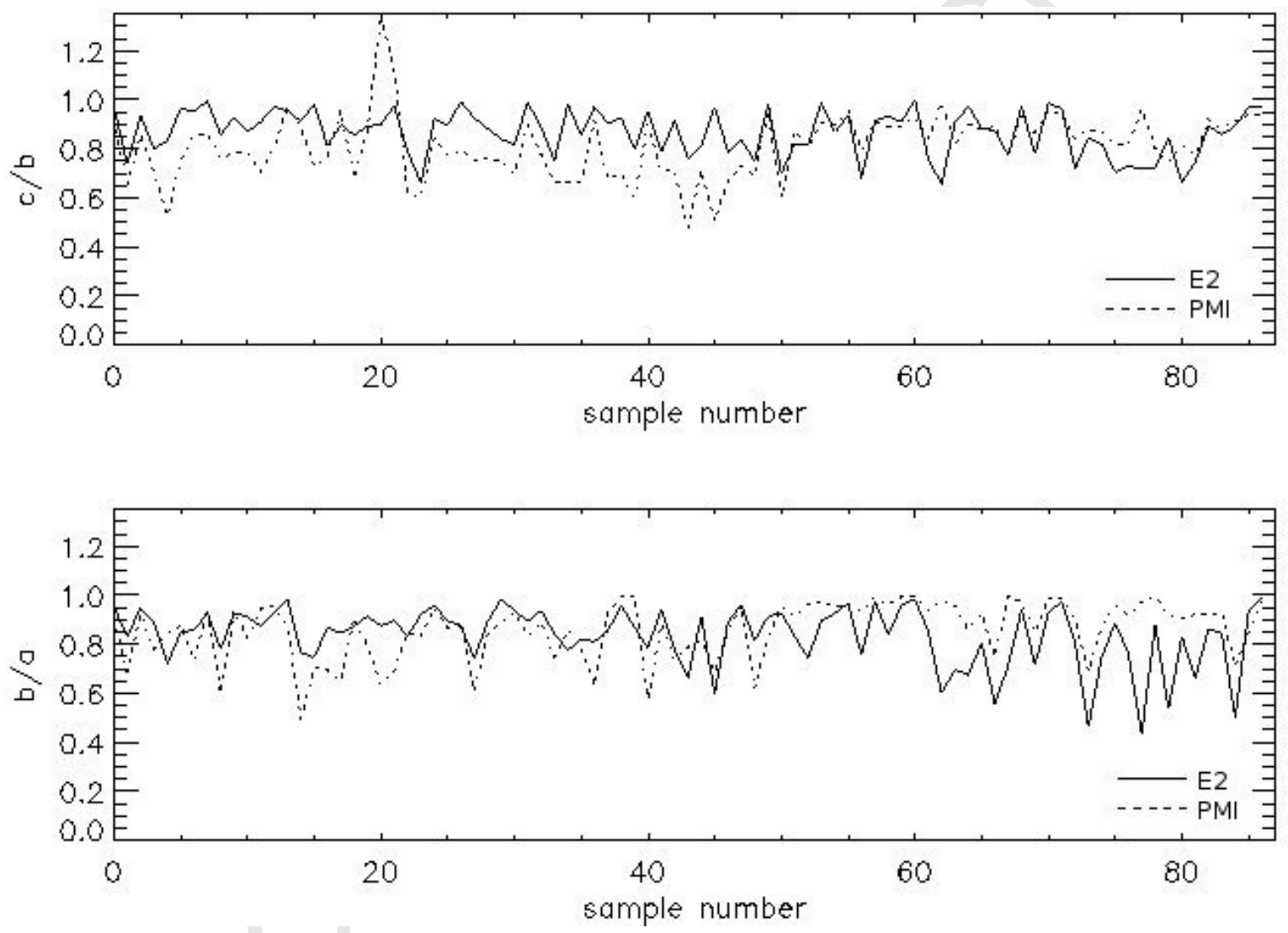


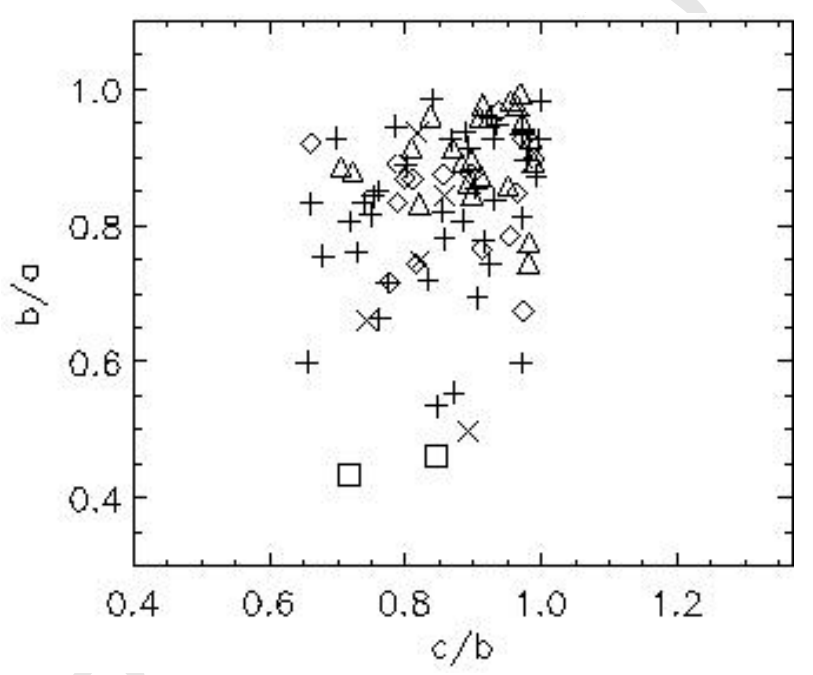




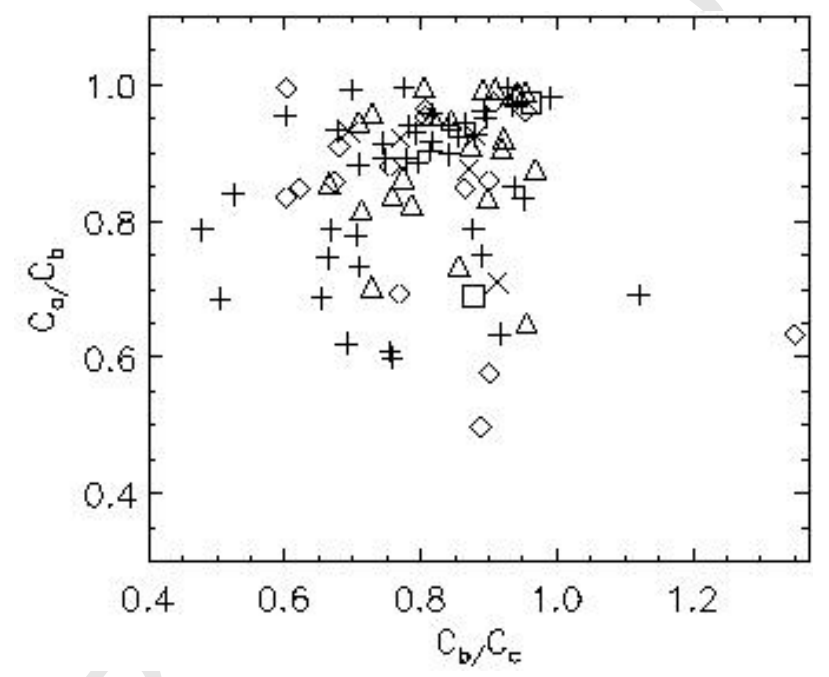




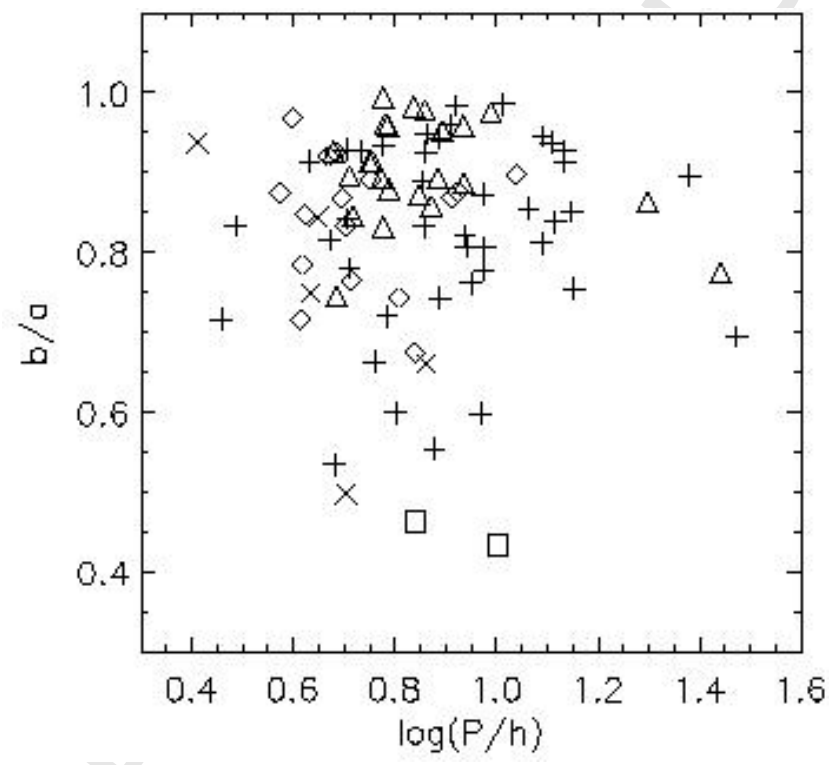




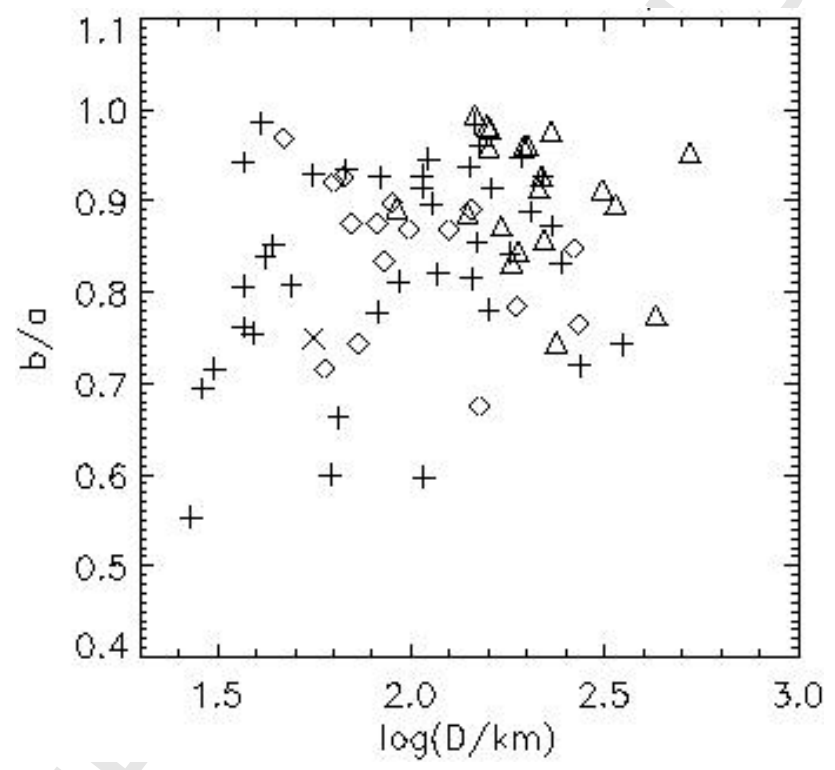



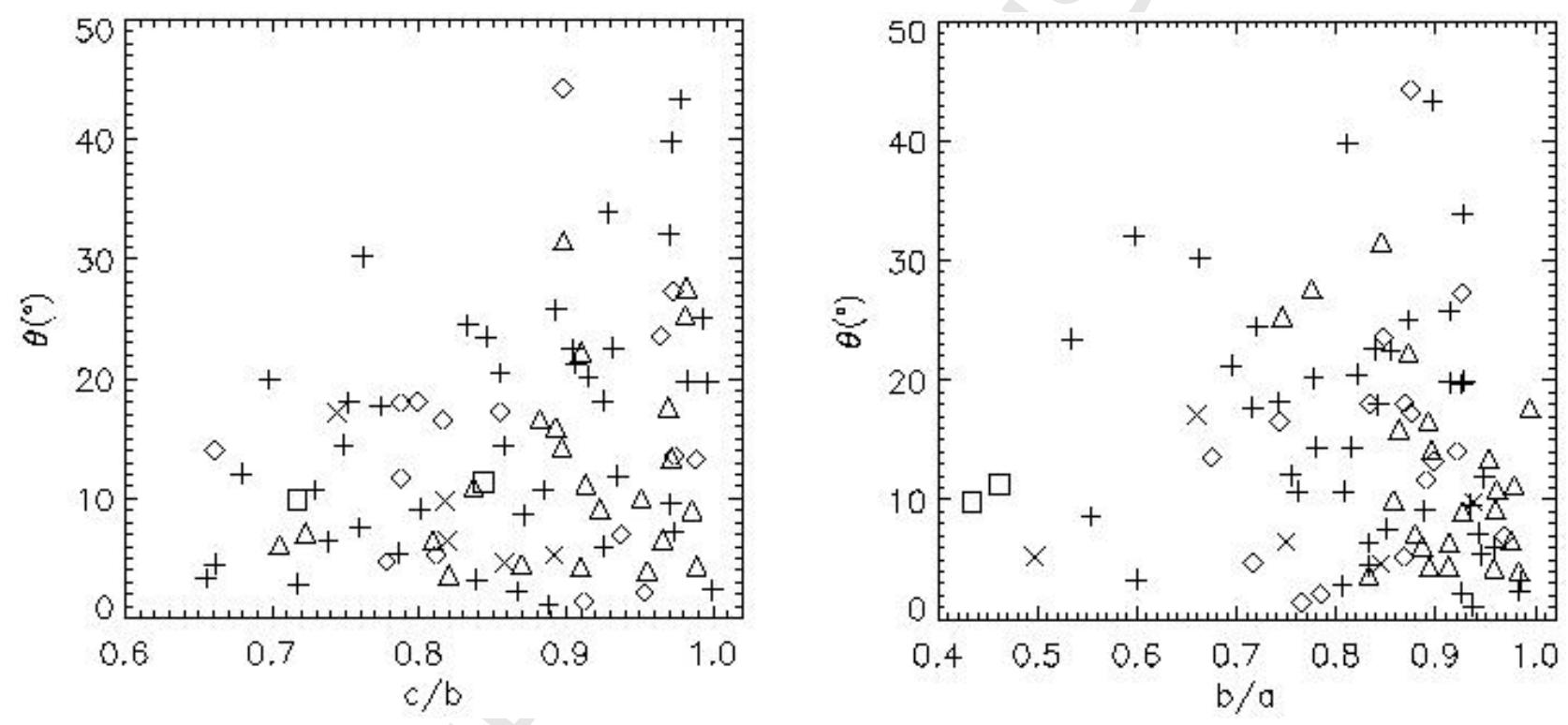

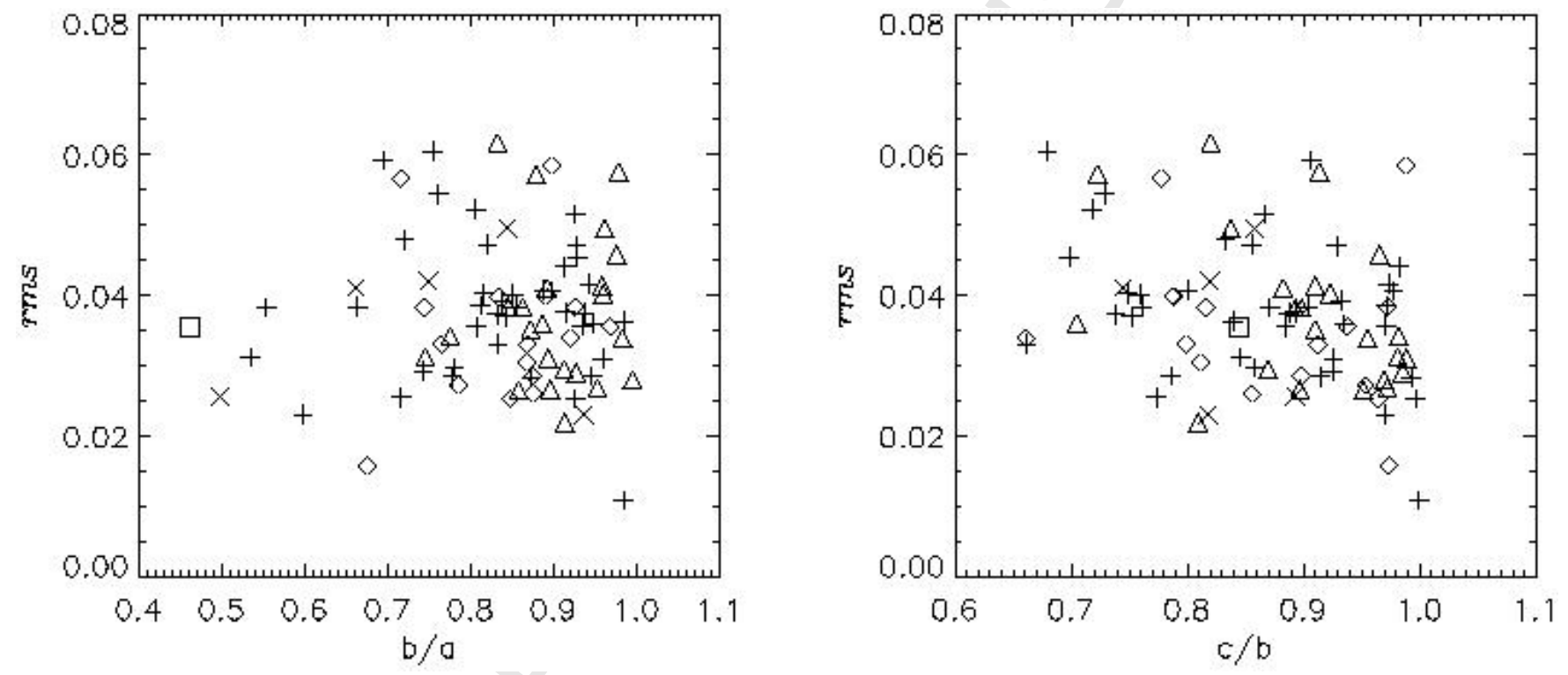


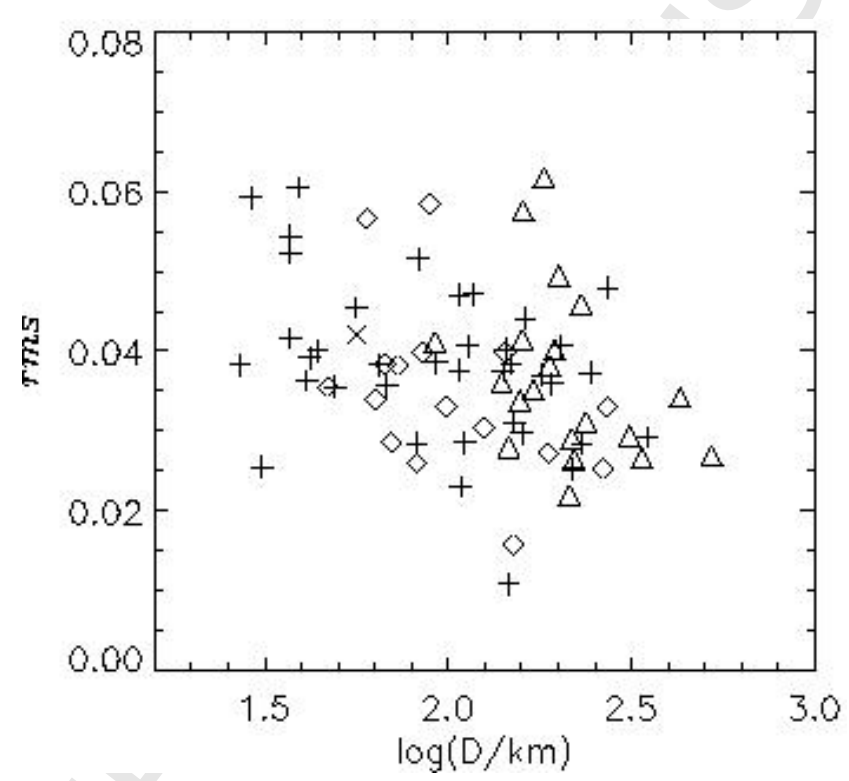




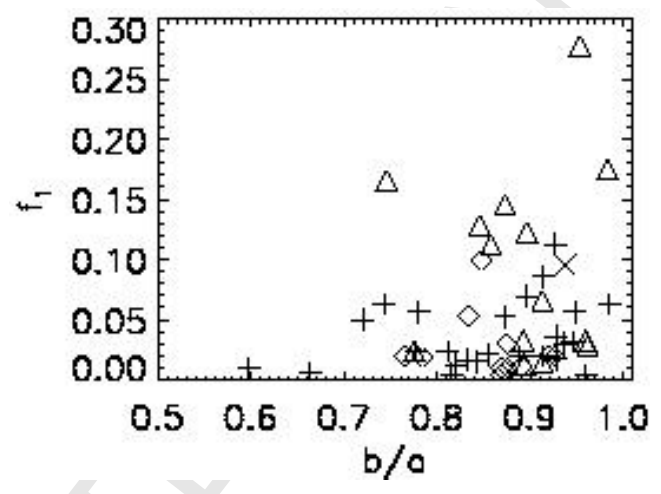




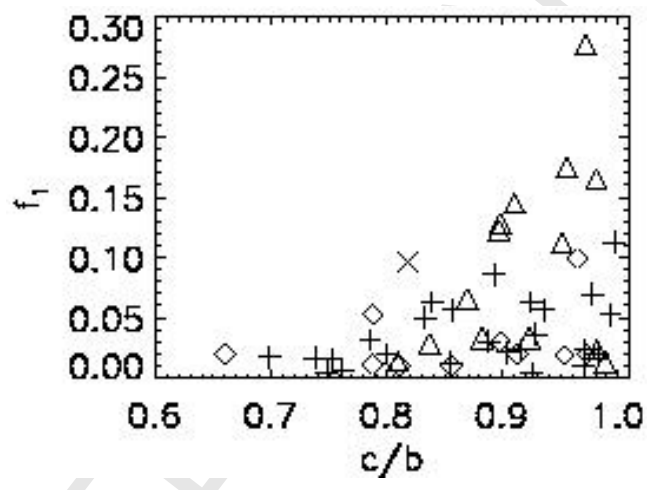




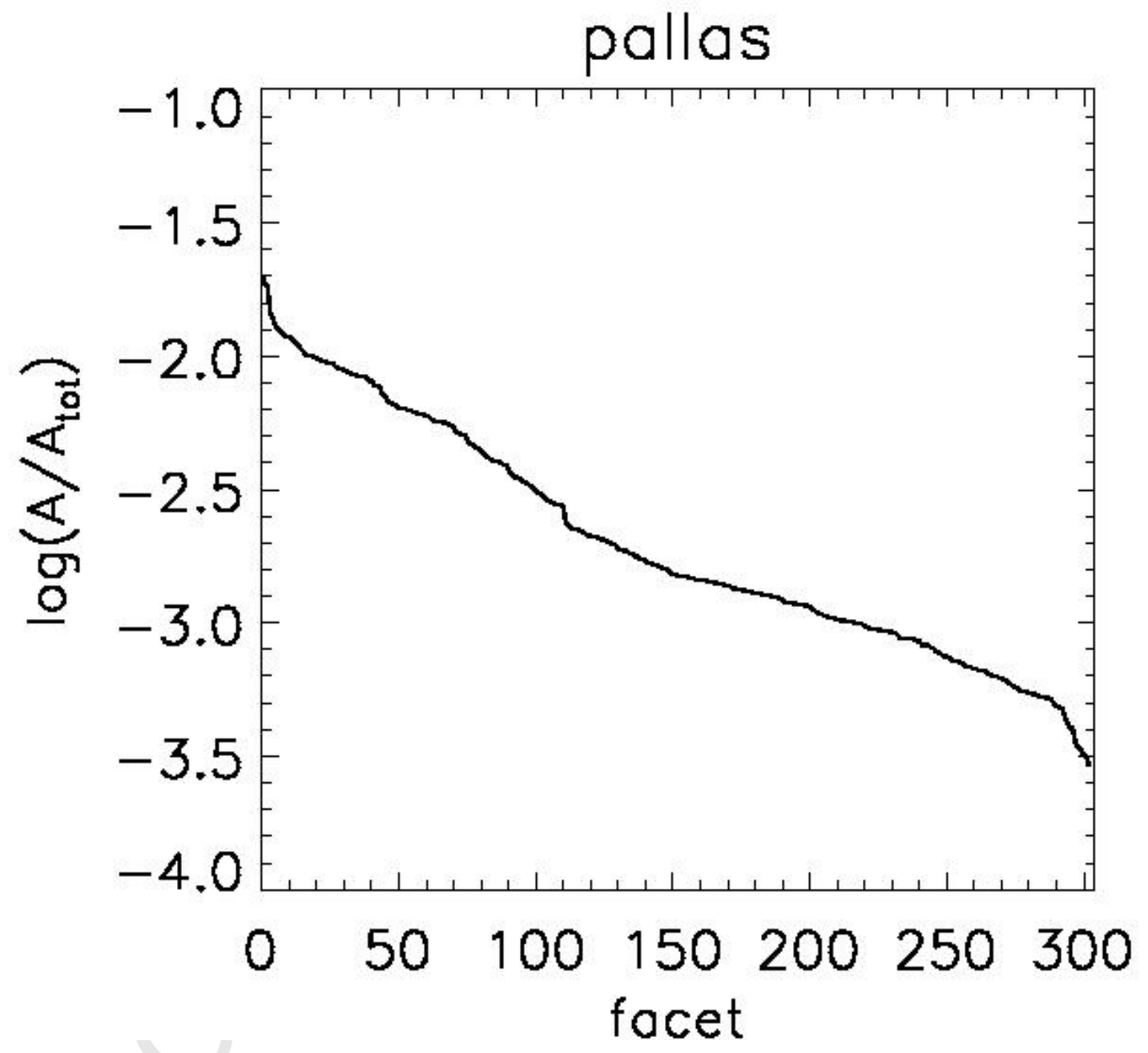




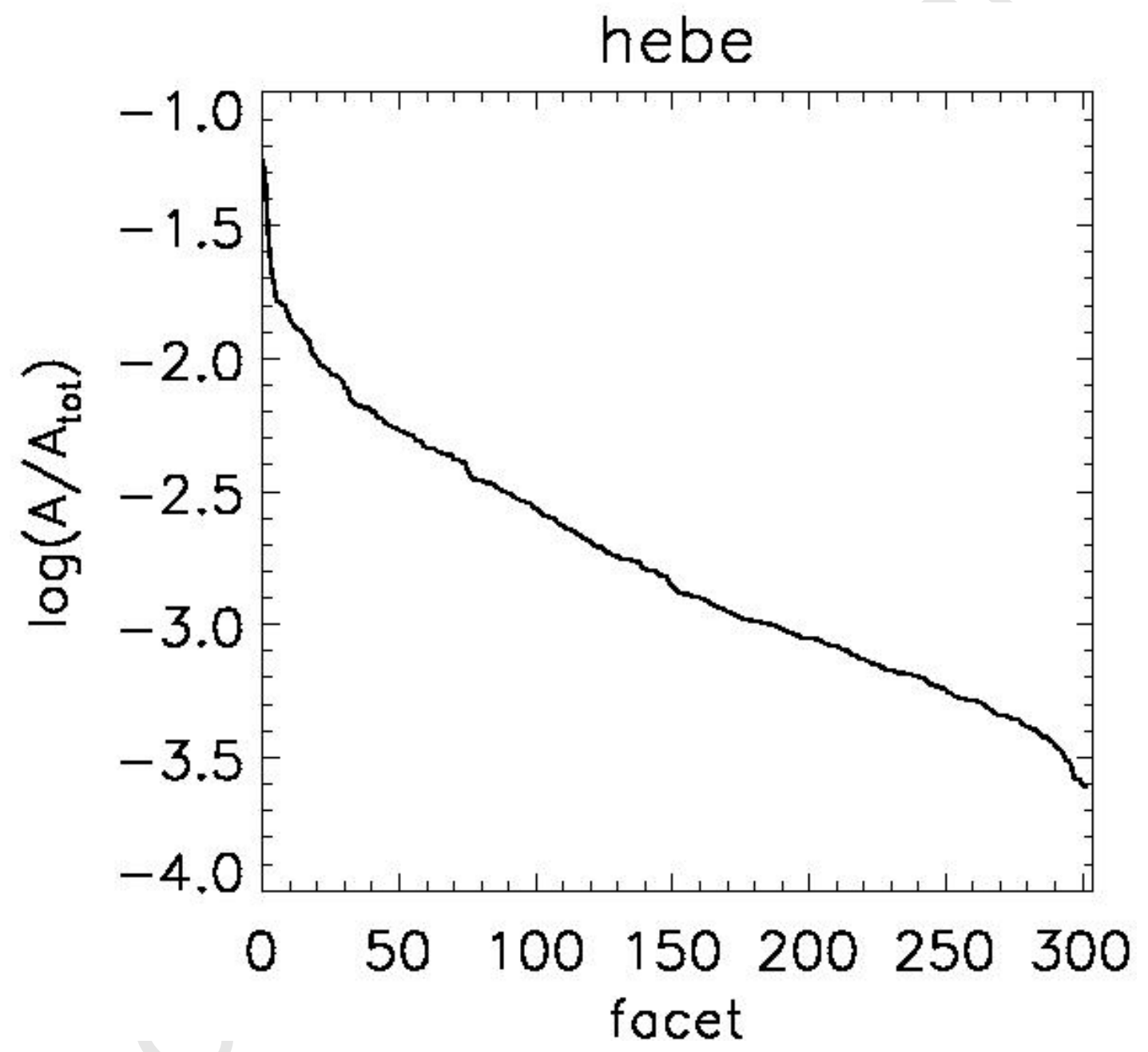




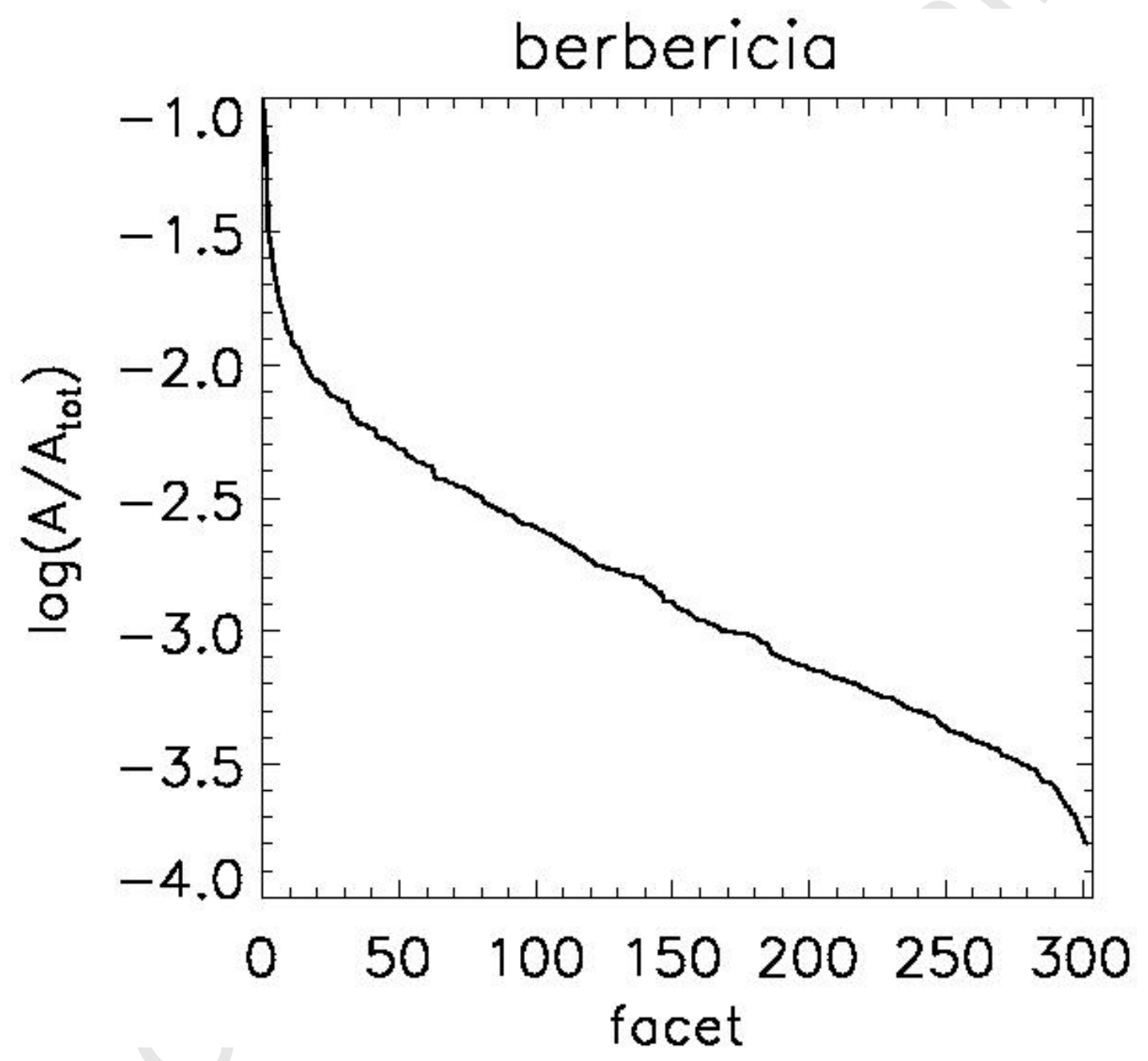




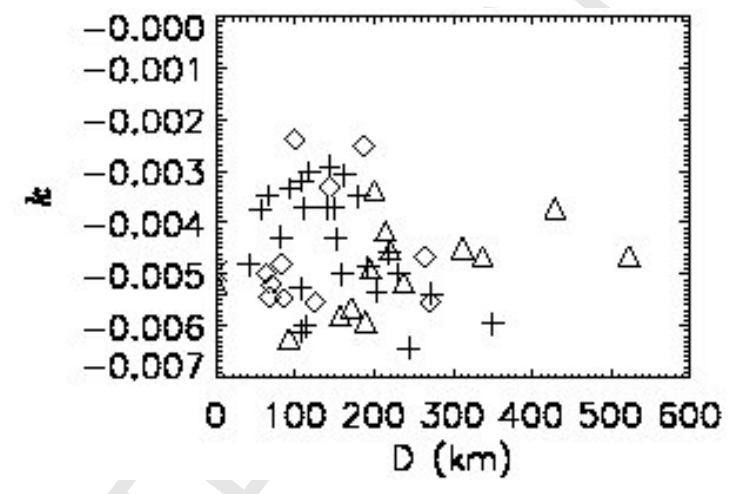

I10268_fig_18 


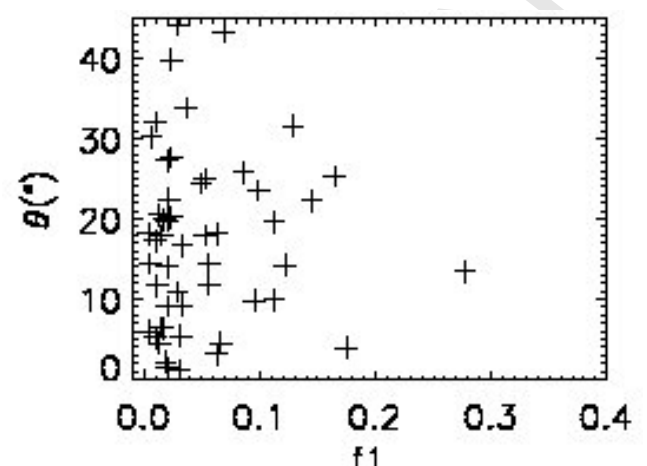

I10268_fig_19 